\title{
Zukunft der Arbeit - Implikationen und Herausforderungen durch autonome Informationssysteme
}

\author{
Rahild Neuburger • Marina Fiedler
}

Eingegangen: 17. April 2020 / Angenommen: 27. August 2020 / Online publiziert: 18. September 2020 (C) Der/die Autor(en) 2020

Zusammenfassung Autonome Informationssysteme (AIS), die lernen, schlussfolgern und entscheiden und damit eigenständig Programme zur Handlung entwickeln, stellen ein zusätzliches Element im Arbeitskontext dar. Je nach Anwendung führen sie dazu, dass sich die Arbeitsteilung zwischen Mensch und Technologie weiter verschiebt. Zwischen den beiden Extrema - Übernahme der Aufgaben alleine durch das AIS bzw. nur durch den Menschen - eröffnet sich ein breites Spektrum an Aufgaben, die in einer neuartigen Form der Arbeitsteilung durchgeführt werden können. Wir beleuchten diese veränderten Arbeitsbedingungen anhand der drei Felder Unternehmensführung, Human-Ressourcen-Management und Organisation und zeigen auf, welche Anforderungen sich hierdurch an die Kompetenzentwicklung des Menschen stellen.

Schlüsselwörter Autonomes Informationssystem · Künstliche Intelligenz · Maschinelles Lernen · Arbeitsteilung · Management · Human-RessourcenManagement · Organisation · Kompetenzentwicklung

\section{Future of Work-Implications and Challenges of Autonomous Information Systems}

Abstract Autonomous Information Systems (AIS), which learn and decide and thus independently develop programs for action, represent an additional element in the working context. Depending on the application, they lead to a further shift in the division of labor between humans and technology. Between the two ex-

R. Neuburger

LMU München, München, Deutschland

M. Fiedler $(\square)$

Universität Passau, Passau, Deutschland

E-Mail: fiedler@1mpi.de 
tremes-assumption of tasks by the AIS alone or only by humans-a broad spectrum of tasks opens up that can be performed in a new form of division of labor. We examine these changed working conditions in the three fields of corporate management, human resources management and organization and show which demands this places on the development of human competence.

Keywords Autonomous information system - Artificial intelligence $\cdot$ Machine learning · Division of labor · Management · Human-resource-management · Organization $\cdot$ Competence development

\section{Einführung}

Die Automatisierung unseres Arbeitslebens ist keine neue Erscheinung. Sie begann in den 1970er Jahren als sogenannte dritte industrielle Revolution mit der Digitalisierung, d.h. der De-Materialisierung von Information in Verbindung mit dem verstärkten Einsatz von Technologien mit dem Ziel der automatisierten Produktion. Der Computer in Verbindung mit Mikrochips und Software zog zunehmend in Fabriken und der Verwaltung ein, indem er menschliche, oftmals manuelle, vorhersagbare Aktivitäten insbesondere des Rechnens und der Buchhaltung unterstützte bzw. schon eigenständig durchführte. Beispiel ist das IBM-System 360, das die Durchführung der damaligen Buchhaltungsprozesse auf eine neue Stufe stellte: Pro Buchungsvorgang wurde eine Lochkarte erzeugt, die wiederum in die EDV-Anlage eingelesen wurde. Aus diesen Buchungs-Lochkarten in Verbindung mit Saldovortragskarten ist der Monatsabschluss automatisiert erstellt worden (Stahlknecht 1982). Das System ersetzte die vormals vom Menschen durchgeführten Buchhaltungsvorgänge. Entsprechend ist der Anteil von Berufen wie Arbeiter in der Produktion, Sachbearbeiter oder auch Vertriebsmitarbeiter in den letzten Jahrzenten gesunken (Autor 2015). Es folgte die wirtschaftliche Nutzung des Internet bzw. des World-Wide-Web. Sie führten zum einen zu einer Automatisierung und Neugestaltung klassischer Einkaufsprozesse (Electronic Procurement) und Verkaufsaktivitäten (Electronic Commerce) (Wirtz 2018), durch die händisch durchgeführte Aufgaben substituiert wurden. Zum anderen entstanden sozio-technische Plattformen, über die die Verteilung, Zuordnung, und Abwicklung von (Kleinst-) Aufträgen an Crowdworker erfolgt, die weitgehend automatisiert gesteuert werden (Malone et al. 2011; Hoßfeld et al. 2012; Picot et al. 2020a; Rai et al., 2019). Als „Internet of Things (IoT)“ diskutiert wurde seit dem Ende der 1990er Jahren (Ashton 1999) die Vernetzung von Dingen, die auf der Basis von Sensorik miteinander kommunizieren und die sich mit Hilfe dieser Daten selbst steuern können. Typische Beispiele sind hier die mobile, dezentrale Ferndiagnose und Fernwartung von Maschinen oder auch die Heizungsanlage, die dem Installateur selbstständig eine Nachricht zukommen lässt, wenn sie droht, auszufallen. Gleichzeitig beschleunigte sich mit der Einführung der Smartphones die mobile automatisierte Nutzung des Internets. Insgesamt führte die exponentielle Leistungssteigerung in Verbindung mit niedrigen Kosten zu unendlichen Rekombinationsmöglichkeiten von automatisierten digitalen Produkten und Services (McQuivey 2014; Brynjolfsson und McAffee 2014, 2017). 
Derzeit befinden wir uns im Rahmen der vierten industriellen Revolution auf dem Übergang von automatisierten zu autonomen Informationssystemen (AIS). In der Managementforschung werden AIS als neue Generation von Informationssystemen definiert, die in der Lage sind, (a) mit der Umwelt zu interagieren, Informationen von außen (auch aus der natürlichen Sprache) oder von anderen Computersystemen zu sammeln; (b) diese Informationen zu interpretieren, Muster zu erkennen, Regeln zu induzieren oder Ereignisse vorherzusagen; (c) Ergebnisse zu erzeugen, Fragen zu beantworten oder Anweisungen an andere Systeme zu geben; und (d) die Ergebnisse ihrer Aktionen auszuwerten und ihre Entscheidungssysteme zu verbessern, um bestimmte Ziele zu erreichen (Ferràs-Hernández 2018).

Beispielhaft erläutern lässt sich dies an den verschiedenen Stufen des automatisierten Fahrens. Hier wird dem Fahrer mit jeder Automatisierungsstufe die Entscheidungsfindung schrittweise abgenommen. Bis zur vierten Stufe - dem hochautomatisierten Fahren - behält er die Kontrolle über die Entscheidung; nicht nur sprichwörtlich hält er das Steuer noch in der Hand. In der 5. Stufe - dem vollautomatisierten oder autonomen Fahren hat der Mensch keine Möglichkeit mehr, zu entscheiden, zu steuern oder einzugreifen, denn dies ist mangels Existenz eines Steuerrades oder von Pedalen nicht mehr vorgesehen (SAE J 3016 2019). Das System lernt basierend auf den zur Verfügung stehenden Daten über Fahrverhalten und entscheidet autonom über die Fahrt. Ähnlich ist es bei AIS, die in Vorschlagssystemen und der Incentivierung und Motivierung von Mitarbeitern zum Einsatz gelangen. So lernen Vorschlagssysteme welche Filme und Serien ein Nutzer unterhaltsam findet und Algorithmen zur Incentivierung lernen, welcher Anreiz wie bei den Mitarbeitern wirkt. Chatbots nehmen Pizza-Bestellungen entgegen und vereinbaren Termine, beantworten Kundenanfragen und bearbeiten Kunden-Beschwerden ohne menschlichen Eingriff, und oft ohne dass der Kunde merkt, dass er mit einem AIS kommuniziert. AIS übernehmen mittlerweile die Vorprüfung von Schadensfällen autonom und filtern diejenigen aus, die von Menschen vorgenommen werden sollen (Peissner et al. 2019). Gleichzeitig interagieren AIS mit anderen AIS, antizipieren deren Aktionen und reagieren autonom darauf. Ein Beispiel sind Maschinen und Anlagen in der Produktion, die autonom in der Lage dazu sind, die über sie laufenden Fertigungsprozesse immer wieder neu und situationsangepasst zu konfigurieren und $\mathrm{zu}$ optimieren, um so flexibel auf neue Aufträge zu reagieren (Dumitrescu et al. 2018). Je mehr und je häufiger sie dies tun, desto besser werden sie üblicherweise dabei, vorausgesetzt die Vorhersagen werden kontinuierlich auditiert. Im Gegensatz zu automatisierten Anwendungssystemen führen AIS Analyse-, Entscheidungs- und Schlussfolgerungsprozesse nicht nur selbstständig und ohne menschlichen Eingriff durch; sie verändern kontinuierlich die Durchführung dieser Prozesse, indem sie sie vor dem Hintergrund ihres Trainings ständig neu justieren und flexibel an die sich verändernden externen Gegebenheiten anpassen.

Die Fähigkeit, kontextfreie Regeln in einen spezifischen Kontext einzubetten und unter all diesen Regeln gewichtet zu handeln, war bislang an den Menschen gebunden. Sie erfordert nicht nur die Kenntnis der geltenden kontextfreien und situativen Regeln, sondern auch die Fähigkeit einzuschätzen, wann welche Regel in welcher Form gilt und dementsprechend geeignet $\mathrm{zu}$ handeln, inklusive der NichtBeachtung geltender Regeln, wenn das die Situation erfordert (Dreyfus und Dreyfus 
1988). Dieser Schritt - vom automatisierten zum autonomen System - lässt sich nun auf Organisationen und die handelnden Akteure übertragen. Ziel ist letztlich, dass autonome Informationssysteme über die Einspeisung von Daten und Ergebnissen lernen, respektive ein Programm zur Handlung entwickeln (Domingos 2015; Tang et al. 2020), wie sie Probleme im organisationalen Kontext lösen können, indem sie eine geeignete Vorhersage treffen. Dabei handelt es sich eher um „enge“ als „generalistische“ AIS, die derzeit entwickelt werden (Broussard 2018). Die Vorhersagemodelle basieren derzeit, anders als beim Menschen, noch nicht auf einem kausalen Verständnis der Zusammenhänge, sondern auf Zusammenhangsinterpretationen. Dennoch können sich AIS in ihrer Aufgabenerfüllung in vergleichsweise engen Anwendungsfeldern aufgrund von Lernen und Training entwickeln. Diese Fähigkeit unterscheidet sie fundamental von automatisierten Informationssystemen (Mertens 1995), bei denen die zugrundeliegenden kausalen Handlungszusammenhänge weitgehend einprogrammiert sind und deren Aufgabe es ist, basierend auf eingegebenen Daten ein Ergebnis zu produzieren (Adler 2019). So können beispielsweise automatisierte Lager- und Beschaffungssysteme individuell an der Geschwindigkeit des Lagerabgangs orientiert nachbestellen, sie sind aber nicht in der Lage, basierend hierauf die Zusammenhänge zu ändern. Diese Fähigkeit, ein Programm zur Handlung zu entwickeln, kann sowohl durch beaufsichtigtes als auch nicht-beaufsichtigtes oder auch bestärkendes Lernen trainiert werden (vgl. Kap. 2).

Durch die Möglichkeiten der AIS zu lernen, vorherzusagen und zu entscheiden wird erstmalig in der Geschichte der Menschheit neues Wissen nicht ausschließlich durch Menschen, sondern auch durch Informationssysteme geschaffen (Domingos 2015). Selbstverständlich lernen AIS aufgrund ihrer Eigenschaften anders als der Mensch (vgl. Kap. 2) und es handelt sich hierbei um einen graduellen Prozess, der noch viele Jahre dauern wird, bis er vollständig Realität wird, und etwa Millionen von Autos autonom auf den Straßen fahren und organisationale Entscheidungen vor allem von AIS getroffen werden.

Auch wenn die Einsatzgebiete von AIS in Organisationen derzeit noch eng sind und sie es in vielen Bereichen noch nicht mit menschlicher Expertise aufnehmen (Strickland 2019), sind sie doch in der Lage, schon jetzt vergleichsweise komplexe Aufgaben zu übernehmen und stellen so zunehmend ein relevantes Element im Arbeitskontext dar (Rai et al. 2019, Zuboff 1988). So sind plötzlich so verschiedene Domänen wie Management, Medizin und Recht vom Einsatz autonomer Informationssysteme betroffen. Dabei erlauben viele AIS derzeit noch den Nutzern, dass die durch den lernenden Algorithmus getroffene Entscheidung abgelehnt werden kann. So kann der Radiologe die Krebsdiagnose durch eine eigene Diagnose ersetzen und der Manager den Bewerbervorschlag des AIS ablehnen. Ihre Eigenschaften des Lernens, autonomen Entscheidens und Vorhersagens greifen jedoch tief und auf eine neue Art in die Arbeitswelt ein, die sich von bisherigen Konsequenzen, wie etwa durch die reine Automatisierung, stark unterscheidet. In der Folge stellt sich die Frage nach einer geänderten Arbeitsteilung zwischen Mensch und AIS, woraus sich neuartige Anforderungen an menschliche Fähigkeiten respektive Kompetenzen und deren Entwicklung ergeben. Ein besseres Verständnis dieser neuen Arbeitsteilung ist wichtig, um zu verstehen, wie AIS in die Arbeitswelt eindringen, welche Art von Aufgaben sie autonom übernehmen und ob und wie sie mit dem Menschen 
zusammenarbeiten, um so die neuen Möglichkeiten für Mensch und Organisation besser zu nutzen.

Vor diesem Hintergrund greift dieser Beitrag zwei Fragestellungen auf: Wie beeinflussen AIS bisher durch den Menschen durchgeführte typische Prozesse in den drei Feldern Unternehmensführung, Human-Ressourcen-Management und unternehmensübergreifende Koordination (Kap. 3) und welche Anforderungen stellen sich hierdurch an die Kompetenzentwicklung (Kap. 4). Ausgangspunkt ist zunächst eine nähere Abgrenzung und Einordnung von AIS als sozio-technischen Systemen (Kap. 2). In Kap. 5 erfolgt ein Ausblick für Forschung und Praxis.

\section{AIS als sozio-technische Systeme}

Obgleich Autonome Informationssysteme (AIS) eigenständig ein Programm zur Handlung entwickeln, durch das sie Probleme im organisationalen Kontext lösen können, hängt der Erfolg von AIS in entscheidender Weise vom Mensch und Aufgabe ab, was sie zu sozio-technischen Systemen macht.

Der Mensch fungiert im Kontext von AIS nicht nur wie bislang als Programmierer oder Anwender; er übernimmt zudem die Rolle des Trainers und beeinflusst so die Handlungsmuster des AIS. AIS lernen völlig anders als der Mensch und verfügen auch meist nicht über einen Körper, in dem sie implizites Wissen speichern. Sie haben aber den Vorteil, dass, wenn sie auf eine Fülle unterschiedlich erfolgreicher menschlicher und nicht-menschlicher Verhaltensweisen zugreifen, damit vorhersagen können, welches Verhalten sich bewährt und welches nicht. Technisch realisiert werden AIS durch die kontinuierliche Erfassung, Extraktion und Analyse von Daten über den eigenen Zustand wie auch der Umgebungsdaten mit Hilfe maschineller Lernverfahren. Die Bausteine des Maschinenlernens sind dabei Repräsentation (z. B. Entscheidungsbäume, Bayesianische Netze/Markovketten, neuronale Netze), Evaluation (z. B. Genauigkeit, Quadrierter Fehler, Wahrscheinlichkeit, Kosten/Nutzen, Marge) und Optimierung (z.B. Kombinatorische Optimierung, Konvexe Optimierung, beschränkte Optimierung) (Domingos 2015). Der Lernvorgang selbst kann dabei folgendermaßen unterschieden werden (Russel und Norvig 2010; Kaplan und Haenlein 2018; Welsch et al. 2018):

- Beaufsichtigtes Imitationslernen (supervised imitation learning):

Diese Art des Lernens basiert auf markierten Daten, die Input-Beispiele für den jeweils korrekten Output beinhalten. Ziel ist, dass das AIS basierend auf diesen richtig gelösten Aufgaben lernt, selbstständig Klassifikationen durchzuführen. Je mehr und bessere Daten verarbeitet werden, desto zutreffendere Ergebnisse können erzielt werden. Typische Beispiele sind die Generierung von Empfehlungen und die automatische Klassifikation von Texten und Bildern. Auch autonomes Fahren basiert auf dieser Art des Lernens (Bansal et al. 2018).

- Nichtbeaufsichtigtes Lernen (unsupervised learning):

Das Training basiert auf nicht-markierten Daten mit dem Ziel, dass das AIS inhärente Strukturen innerhalb der Daten entdeckt und clustert. Die Gruppierung von Elementen in gleichartige Kategorien, bei denen weder die Struktur dieser Cluster 
noch die Anzahl vorab bekannt ist, fällt in diese Lerngruppe. Typische Anwendungsbeispiele sind die Spracherkennung durch Siri und Alexa.

- Bestärkendes Lernen (reinforcement learning):

Das Training basiert auf bestärkendem Feedback. So führt das System eine Aktion durch und wird direkt belohnt oder bestraft. Beispiel ist ein AIS, das zum Ziel hat, Pac-Man spielen zu lernen, indem es nur weiß, dass es sich hoch, runter, links und rechts bewegen kann und, dass das Ziel darin besteht, den Punktestand zu maximieren. Microsoft nutzt bestärkendes Lernen, um Schlagzeilen auf MSN.com auszuwählen, indem das AIS mit einer höheren Score belohnt wird, wenn mehr Besucher auf den ausgewählten Link klicken (Kaplan und Haenlein 2018). Weitere Beispiele sind der Einsatz in der Robotik oder in der Logistikplanung.

Alle drei Arten von Maschinenlernen basieren auf Trainingsdaten. Broussard (2019) gibt folgendes Beispiel für den Bereich von Kreditkartenanbietern:

Nehmen wir an, dass meine Trainingsdaten ein Datensatz von hunderttausend Kunden von Kreditkartenunternehmen sind. Der Datensatz enthält die Daten, die man von einem Kreditkartenunternehmen für eine Person erwarten würde: Name, Alter, Adresse, Kreditwürdigkeit, Zinssatz, Kontostand, Name(n) aller Mitunterzeichner des Kontos, eine Liste der Gebühren und eine Aufzeichnung der Zahlungsbeträge und -daten. Nehmen wir an, wir wollen, dass das ML-Modell vorhersagen kann, wer seine Rechnung wahrscheinlich verspätet bezahlen wird. (...) Die Trainingsdaten haben eine Spalte, die angibt, wer in dieser Gruppe von hunderttausend Personen seine Rechnungen verspätet bezahlt hat.

Wir teilen die Trainingsdaten in zwei Gruppen von je fünfzigtausend Namen auf: das Trainingsset und die Testdaten. Dann führen wir einen maschinellen Lernalgorithmus mit dem Trainingsset aus, um ein Modell, (...) zu konstruieren, das vorhersagt, was wir bereits wissen. Dann können wir das Modell auf die Testdaten anwenden, um die Vorhersage des Modells für die Kunden zu sehen, die wahrscheinlich zu spät bezahlen werden. Schließlich vergleichen wir die Vorhersage des Modells mit dem, von dem wir wissen, dass es wahr ist den Kunden in den Testdaten, die tatsächlich verspätet bezahlt haben. Dadurch erhalten wir ein Ergebnis, das die Präzision des Modells (...) misst. Wenn wir als Modellhersteller entscheiden, dass die Präzision (...) des Modells hoch genug ist, können wir das Modell auf echte Kunden anwenden (Broussard 2019, S. 7).

Oft liegen Algorithmen und insbesondere AIS in ihrer Vorhersage genauer als der Mensch (Dietvorst et al. 2014, 2016), interessanterweise nicht nur bei sachlich-neutralen Themen, sondern selbst bei emotionalen Themen wie der Vorhersage, welche Witze Menschen lustig finden. Yeomans et al. (2019) finden heraus, dass AIS treffsicherer als Freunde und Familienmitglieder vorhersagen können, welchen Witz ein Mensch lustig findet. Dennoch ist beim Menschen häufig sogenannte Algorithmusaversion festzustellen, die zur Ablehnung der AIS-Vorhersage führen. Berechtigt ist diese im Falle von Verzerrungen (z. B. in den Trainingsdaten, im spezifizierten Modell oder auch beim Menschen, der als Programmierer und Trainer fungiert). 
So gibt es zahlreiche Beispiele für Verzerrungen sowie falsche und verzerrte Identifikation und Klassifizierung durch AIS. So wurden Katzen als Hunde, Hunde als Wölfe und, weit bedeutsamer und folgenschwerer, Menschen aufgrund ihrer Ethnizität fehlerhaft als potenziell straffällig (Hatmaker 2020) oder nicht behandlungswürdig (Obermeyer et al. 2019) eingestuft. Hier stellt sich die Frage, was das AIS eigentlich klassifiziert hat (z.B. Wolf/Hund oder Schnee/Sand). Zu den bekanntesten Negativ-Beispielen im Managementbereich gehört die Aussortierung weiblicher Bewerbungen bei Amazon für Stellen als Softwareentwickler und andere technische Aufgaben, da das AIS auf Grund der existierenden tatsächlichen Einstellungsdaten über einen Zehn-Jahres-Zeitraum, die zum Training genutzt wurden, geschlussfolgert hat, dass weibliche Mitarbeiterinnen ungeeignet sind (Dastin 2018). Diese Verzerrung liegt weniger in der Klassifizierung als in der bewussten oder unbewussten menschlichen Verzerrung bei Einstellungsprozessen, die beispielsweise darin begründet sein kann, dass man lieber Leute einstellt, die einem ähnlich sind (Homophilie) oder von denen man sich unbewusst bessere Leistung erwartet. Weitere Beispiele für nutzlose und teilweise auch gefährliche Empfehlungen durch AIS können in fehlerhaften Empfehlungen durch „Watson for Oncology“ für Krebsbehandlungen (Ross und Swetlitz 2018) und dem korrumpierten Microsoft AI-Chatbot Tay gesehen werden, der über das Training mit ,relevanten öffentlichen Daten“ Rassismus, Frauenfeindlichkeit und Antisemitismus gelernt hat (Blier 2020). Im Kontext mit AIS wurde hier zudem vielfach über ethische Fragen und Vorurteile des Menschen diskutiert, die über das Training Einfluss nehmen auf die Vorhersagen und Handlungen des AIS. Zu den bekanntesten Beispielen gehört das Trolley Dilemma (vgl. z. B. https://strongbytes.ai/the-trolley-dilemma-in-ai/), bei dem das AIS durch entsprechende Handlung die Wahl hat, einen außer Kontrolle geratenen fahrenden Zug, der auf fünf Menschen zufährt, dahingehend zu stoppen, dass es die Weichen so stellt, dass ein Mensch, der auf dem umgeleiteten Gleis steht, opfert. Je nachdem, ob der Programmierer oder Trainer des AIS eher utilitaristisch (fünf statt einem Menschenleben retten) oder deontologisch (aktives Tötungsverbot, Zug rollt weiter auf seinem ursprünglichen Gleis und tötet die fünf Menschen) eingestellt ist, wird das AIS entsprechend beeinflusst (Ramaprasad, J. et al. 2020). Ein weiterer großer menschlicher Einflussfaktor auf den Einsatz von AIS in Organisationen dürfte das Vertrauen spielen, das Menschen diesen Systemen entgegenbringen. Glikson und Woolley (2020) kommen basierend auf einer Übersicht der AI-Forschung der letzten 20-Jahre zu dem Befund, dass die physische und visuelle Präsenz, Transparenz, Verlässlichkeit und Unmittelbarkeit des AIS Einfluss auf die Entwicklung kognitiven Vertrauens spielt. Anthropomorphisierung des AIS wirkt zudem auf die Entwicklung emotionalen Vertrauens beim Mensch. Dabei entwickelt sich im Durchschnitt Vertrauen gegenüber Roboter-AIS ähnlich wie gegenüber Menschen, es startet niedrig und je nach direkter Erfahrung steigt es. Bei virtueller und embedded AIS ist es umgekehrt. Hohes anfängliches Vertrauen sinkt durch Erfahrung mit dem AIS. Niedriges Vertrauen in AIS führt üblicherweise zu niedrigem Einsatz dieser Systeme, sofern eine Handlungsfreiheit des Mitarbeiters besteht. Zudem gibt es eine Reihe von menschlichen Moderatoren, die den AIS-Einsatz beeinflussen. Dazu gehören u.a. die Selbstwahrnehmung der eigenen Fähigkeiten und Kompetenzen und der eigene Expertisegrad. 
An all diesen Beispielen ist zu erkennen, dass das AIS auf die gegebenen Daten und das Training angewiesen ist, um entsprechende Vorhersagen zu treffen und kein kausales Verständnis der Zusammenhänge hat. Anders als beim menschlichen Experten, bei dem man sich daran gewöhnt hat, dass er seine Diagnose nicht erklärt, da man ihm aufgrund seiner Expertise ein gewisses kausales Verständnis unterstellt, ist das bei AIS, insbesondere dann, wenn das AIS mit Mitteln der staatlichen oder privaten Machtausübung verbunden ist (z.B. Ablehnung des Kreditkartenantrags, Straffälligkeitsprognose aufgrund von racial profiling, Einreiseverbot u. ä.) nicht akzeptabel. Gesetze wie die Datenschutzgrundverordnung in der EU oder der Fair Credit Reporting Act in den USA sind erlassen worden, um die Nachvollziehbarkeit und Erklärbarkeit der Entscheidung eines AIS zu gewährleisten und etwaige Verzerrungen zu erkennen. Dabei ist es wichtig zu definieren, wie und zu welchem Ausmaß AIS erklärbar zu sein haben. Geht es ausschließlich um die Offenlegung einzelner Variablen, um deren Verbindung oder um das gesamte Modell mit allen Gewichtungen? Wie gut müssen die Menschen ausgebildet sein, die mit diesen Systemen arbeiten? Wie nachvollziehbar die ML-Elemente? Wer ist berechtigt zur Prüfung des AIS? Kritiker dieser Richtlinien zur Nachvollziehbarkeit und Erklärbarkeit thematisieren insbesondere das Manipulations- und Gamingpotenzial, das durch die Erklärbarkeit entsteht. Wenn jeder Nutzer, Designer und Auditor den gesamten Algorithmus nachvollziehen kann, ist entsprechende Anpassung des Verhaltens zu erwarten, was wiederum zur Änderung des Algorithmus führt, da dieser in der Lage ist, eigenständig ein Programm zur Handlung zu entwickeln. Befinden wir uns in einem Datenkomplexen Bereich mit geringer Umweltkomplexität, so ist fragwürdig, ob der Mensch dieses Katz-und-Maus-Spiel je gewinnen kann.

Entscheidend für die Einsatzmöglichkeiten und letztlich auch Implikationen von AIS auf Arbeit sind zum einen die ausgewählten maschinellen Bausteine und Lernverfahren; zum anderen Art und Inhalt der dem Training zugrundeliegenden Daten und die dazugehörige Infrastruktur. Und genau in der Kombination von Daten und Lernverfahren liegt nicht nur ein Unterschied zu automatisierten Informationssystemen, sondern auch eine wesentliche Herausforderung und Grenze von AIS. Denn ob ein AIS erfolgreich eingesetzt werden kann, hängt abgesehen davon, ob die juristische Regulierung wie etwa Mitbestimmung und Datenschutz als auch ethische Überlegungen es ermöglichen, entscheidend zum einen davon ab, ob das zugrundeliegende maschinelle Verfahren stimmig zu dem zu bewältigenden Problem ist. Zum anderen v. a. aber auch von der Verfügbarkeit der für das maschinelle Verfahren erforderlichen Daten als auch deren Qualität für das Training.

All diese Beispiele verdeutlichen nochmals das Kernprinzip von AIS: sie basieren auf Methoden des maschinellen Lernens und werden mit Hilfe von Daten und kontinuierlichem Training in die Lage versetzt, ein Programm zur Handlung zu entwickeln. Sind die verwendeten Trainingsdaten und das Training verzerrt und das Modell beispielsweise overfitted und nicht nachvollziehbar, sind die so ermittelten Vorhersagen fragwürdig bis falsch. Gleichzeitig ermöglicht ein konsequentes Audit von AIS-Vorhersagen und den zugehörigen Elementen aber auch in noch nicht gekannter Weise die Offenlegung, Identifikation und Hinterfragung menschlicher Verzerrungen in der Entscheidung, wie das Beispiel von Amazon sehr eindringlich verdeutlicht. Erst durch die Prüfung der AIS-Empfehlung sind die Verzerrungen 
im menschlichen Entscheidungsverhalten offengelegt worden. $\mathrm{Zu}$ den bekanntesten menschlichen Verzerrungen bei Auswahlentscheidungen gehören der „Homophilie“und der „Unconscious“-Effekt, die beide dazu beitragen, dass man insbesondere Kandidaten und Merkmale bevorzugt, die als ähnlich wahrgenommen werden, auch wenn man sich dessen nicht unbedingt bewusst ist (Fiedler et al. 2011).

\section{AIS und ihre Implikationen auf Arbeit}

Im Folgenden zeigen wir auf, wie AIS die Arbeitsteilung in und von Organisationen beeinflussen, um so den Einfluss auf menschliche Kompetenzen und deren Entwicklung zu identifizieren. Um die Vielfalt an unterschiedlichen Einsatzmöglichkeiten aufzuzeigen, diskutieren wir dies anhand der drei Bereiche Unternehmensführung, Human Ressourcen-Management sowie Konfiguration und Koordination von Prozessen und Strukturen.

\subsection{Unternehmensführung und AIS - eine neue Form der Koexistenz?}

Typischerweise erstrecken sich Führungsaufgaben von inhaltlich-unternehmensbezogenen Aufgaben auf strategischer und operativer Ebene bis hin zu mitarbeiterbezogenen Führungsaufgaben, zu denen insbesondere Einflussnahme und Motivation zählen. Die zugrundeliegenden Tätigkeiten sind ähnlich: Erfassen von Informationen, Interpretieren, Schlussfolgern, Entscheiden, Steuern, Kontrollieren, Revidieren, Kommunizieren, Verantwortung übernehmen etc.

Sie sind z.T. strukturiert und entsprechen einer gewissen ,wenn-dann-Logik“ (z.B. ,wenn der EBIT einen vorgegebenen Wert x unterschreitet, müssen bestimmte Maßnahmen eingeleitet werden“). Zum Teil sind sie aber auch unstrukturiert und basieren auf Kreativität, Intuition, Gewissenhaftigkeit, emotionaler und sozialer Intelligenz, einer ganzheitlichen Situationserfassung oder auch auf der kommunikativ angemessenen Integration verschiedener Meinungen und Perspektiven. Beispielsweise erfordert die Entscheidung über die Weiterentwicklung des Geschäftsmodells in Folge von Profitabilitätsdruck, dass Manager sowohl ökonomische Logik als auch oft weniger klar strukturierte Aufgaben wie verantwortungsvolles, nachhaltiges Handeln gegenüber den Mitarbeitern in ihre Entscheidung und Verhaltensweisen integrieren.

Hinzu kommt, dass es wenig veränderliche Führungsaufgaben gibt, die sich in regelmäßigen Zeitabständen wiederholen; andere Führungsaufgaben wiederum ad hoc auftreten und schnelles Agieren oft auch auf der Basis einer schlechten Informationsund Datenlage erfordern. Beispielsweise führen neue Marktteilnehmer und plötzlich veränderte Kundenpräferenzen dazu, dass Führungskräfte Ziele, Kenngrößen und Verhaltensweisen anzupassen haben, um am Markt zu bestehen.

Führungsentscheidungen sind zudem in der Regel komplex, wobei diese Komplexität sowohl die Beherrschung einer Vielzahl an kontextfreien Daten und Informationen und deren Analyse, als auch die Erfassung der zugrundeliegenden situativen Umwelt- und Kontextparameter erfordert. Von besonderer Bedeutung ist dabei einerseits die geeignete Einbettung der kontextfreien Regeln in die jeweilige Situation, 
als auch die individuelle Gewichtung der Faktoren. Dies kann auch ein Abweichen von den kontextfreien oder situationalen Regeln bedingen (Dreyfus und Dreyfus 1988).

In diesem - zugegebenermaßen etwas vereinfacht dargestellten - Spektrum an Führungsentscheidungen, die sich einerseits zwischen gering und hoch strukturiert als auch gering und hoch veränderlich bewegen und zum dritten die Beherrschung unterschiedlicher Arten an Komplexität erfordern, können Führungskräfte nun Aufgaben an AIS verlagern.

$\mathrm{Zu}$ vermuten ist zunächst, dass sich Führungsaufgaben, die sich durch hohe Strukturiertheit, geringe Veränderlichkeit sowie Daten- und Analysekomplexität auszeichnen und damit vorhersagbar sind, für die autonome Abwicklung durch AIS sprechen. Je mehr AIS hier eingesetzt werden und je größer dadurch die zugrundeliegende Fallbasis wird, desto mehr werden sie trainiert und desto besser sind sie in der Lage, die zugrundeliegenden Führungsaufgaben ohne Eingriff der Führungskräfte durchzuführen, sofern ihre Vorhersagen wie gesagt kontinuierlich auditiert werden. So setzt z. B. das Unternehmen Bridgewater Associates unter dem Namen PriOS ein AIS ein, das alltägliche Management-Aufgaben alleine übernehmen soll. Dabei soll es von den Entscheidungsstrukturen des Gründers sowie der Top-Manager lernen, um ihre Prinzipien bei den Entscheidungen zugrunde zu legen (Scheelen 2019). Wie schon zuvor am Amazon Beispiel verdeutlicht, liegt hier die besondere Herausforderung in der Prüfung der dem AIS zu Verfügung gestellten Daten zum Lernen seiner Entscheidungen sowie dem Training des Systems. Sind diese Trainingsdaten und das Training aufgrund der zugrundeliegenden menschlichen Entscheidungen verzerrt, so werden auch die AIS-Entscheidungen verzerrt sein.

An ihre Grenzen stoßen AIS zudem derzeit dann, wenn es um nicht strukturierte oder ad-hoc-auftretende Führungsentscheidungen geht, die insbesondere auf Grund der Kontextkomplexität nicht vorhersagbar sind. Typische Beispiele sind Krisensituationen, deren Ursache auf nicht vorhersehbare und bisher nicht bekannte Parameter zurückzuführen sind. Das Bild des AIS, das in einer Krisensituation die Produktion bestimmter Lebensmittel stoppt, da es die plötzlich stark ansteigende Nachfrage als „kann nicht so stimmen“ interpretiert, mag hier als Beispiel dienen. Eine menschliche Führungskraft mit Expertise und entsprechendem Verständnis der kausalen Zusammenhänge hätte die Situation individuell erfasst und die Zahlen entsprechend als korrekt interpretiert. Ähnliches gilt - zumindest vor dem Hintergrund des jetzigen technologischen Entwicklungsstandes - für mitarbeiterbezogene Führungsaufgaben wie Einflussnahme, Kohäsion, Wertevermittlung als auch Motivation. Gerade hier lassen sich Aufgaben und Entscheidungen erkennen, die unstrukturiert sind, ad hoc auftreten und v. a. Empathie, Intuition, Erfassen der Komplexität und multiperspektivischen Kontextbezug erfordern und damit schlecht planbar sind. Je weniger strukturiert, hoch veränderlich und durch Kontextkomplexität charakterisiert und damit unvorhersehbar die Führungsaufgaben sind, desto weniger macht es derzeit noch Sinn, diese Aufgaben durch AIS zu realisieren. Hierin liegen die Stärken des Menschen (Rai et al. 2019). Aber auch hier ist festzustellen, dass AIS zunehmend in diese Bereiche eindringen. Incentivierung und Teambildung erfolgt schon heute bei technischen, plattformbasierten Geschäftsmodellen über AIS (Rai et al. 2019; Gregory et al. 2020; Ihl et al. 2020). Auch emotionale Ansprache ist 
durch AIS lern- und einsetzbar, was man an der Beliebtheit von anthropomorphisierten Robotern und Chatbots sieht.

Zwischen den beiden Extrema - Übernahme der Führungsaufgaben ausschließlich durch das AIS bzw. ausschließlich durch die menschliche Führungskraft - eröffnet sich ein breites Spektrum an Führungsaufgaben, die in einer neuartigen Form der Arbeitsteilung durchgeführt werden können. Besonders interessant ist der Bereich, bei dem die menschlichen Führungsfertigkeiten durch AIS unterstützt respektive augmentiert werden und diese damit auf ein neues Fähigkeitsniveau heben (Raisch und Krakowski 2020). Ein Beispiel könnte die durch das AIS vorgenommene statistische Auswertung von Kundendaten sein, bei der die Führungskraft (im Fall von erklärbarem AIS) nicht nur das Ergebnis erhält, sondern bei der auch der dahinterliegende Schlussfolgerungsprozess verdeutlicht wird, so dass die Führungskraft dadurch neues Wissen aufbaut und befähigt wird, in andere Richtungen zu denken.

Das AIS unterstützt hier also nicht nur den Menschen durch effizientere oder auch bessere Entscheidungen; dieser lernt durch die Nutzung des AIS und kann die Handlungsmuster des AIS beispielsweise nutzen, um neuartige Lösungen zu entwickeln. Dieser augmentierte Lernprozess führt dazu, dass Führungsaufgaben im Team aus Mensch und AIS zielführender durchgeführt werden können. Mensch und AIS stellen hier keine Konkurrenten dar. Vielmehr agieren sie in kooperativer Koexistenz, indem sie ihre jeweiligen Stärken problemorientiert verknüpfen und voneinander profitieren. Liegt die Stärke des AIS in der Erstellung komplexer Modelle, die auf großen Datenmengen angesetzt werden und neue Strukturen identifizieren als auch das Wissen verschiedener menschlicher Trainer verbinden kann, liegt die Stärke des Menschen darin, diese Vorhersagen vor dem Hintergrund seines kausalen Verständnisses, seiner Intuition und seiner Imagination abzuwägen und auf dieser Basis Entscheidungen zu treffen. Gleichzeitig sammelt der Mensch so Erfahrung und ist dadurch in der Lage, bestimmte Sachverhalte zukünftig noch besser einzuschätzen.

Im Ergebnis gibt es Führungsaufgaben, die das AIS autonom durchführt; Führungsaufgaben, die beim Menschen bleiben sowie Führungsaufgaben, die Mensch und AIS gemeinsam durchführen und dabei voneinander profitieren. Der Mensch durch Aufbau von Erfahrung und das AIS durch zusätzliches Training. Spannend in diesem ,hybriden“ Bereich der Augmentierung ist, wie die von Mensch und AIS jeweils durchgeführten Aufgaben ineinandergreifen, wer Entscheidungen und Schlussfolgerungen der AIS verantwortet und ob ein kontinuierliches Training von AIS dazu führt, dass diese immer mehr Aufgaben von Führungskräften übernehmen werden. Sind AIS hierzu in der Lage, entsteht eine neue Dimension der Arbeitsteilung zwischen Mensch und Maschine. Führungskräfte können bisher dem Menschen vorbehaltene Tätigkeiten wie Schlussfolgern, Vorhersagen oder Entscheidungen treffen an AIS delegieren. Hierdurch entstehen zeitliche und mentale Freiräume, um sich mit den z. T. hoch-komplexen Herausforderungen der Führungskraft zu beschäftigen. Gleichzeitig erfordert genau diese neuartige Form der Arbeitsteilung neuartige Kompetenzen von den Führungskräften, die weit über reine Mensch-Maschine-Interaktionskompetenzen hinausgehen (vgl. Abschn. 4). 


\subsection{Human-Ressourcen-Management durch AIS?}

Human-Ressourcen-Management umfasst so unterschiedliche Aufgaben wie Bestimmung der Arbeitsinhalte, -prozesse, und-strukturen sowie der damit einhergehenden Aufgaben wie etwa Personalauswahl, Motivations- und Anreizsystemgestaltung als auch Talent Management, Kompetenzentwicklung und People Strategy.

Primär tangiert ist zunächst der administrative Bereich, in dem schon lange viele gering veränderliche und gut strukturierte Routineprozesse der Personalverwaltung durch automatisierte Informationssysteme durchgeführt werden können. Typische Beispiele sind reine Verwaltungsprozesse wie z.B. die Abwicklung der Reisekosten, Lohnbuchhaltung und Urlaubsplanung. Neu ist jetzt, dass AIS selbstständig die Suche und die Ansprache externer Bewerber übernehmen. Beispiel ist das „Programmatic Job Advertising“, bei dem Einkauf, Platzierung und Optimierung von Stellenanzeigen durch AIS erfolgen. Dieses System verteilt ohne menschlichen Eingriff Stellenanzeigen datenbasiert über ein weitreichendes Personal-Marketing-Netzwerk aus Jobsuchmaschinen, sozialen Medien und Bannerwerbung an aktive und passive Interessenten in der Zielgruppe. Bei Interesse erfolgt eine direkte Weiterleitung auf die unternehmenseigene Karriereseite, über die sofort die Bewerbung verschickt werden kann. Durch die dabei gesammelten Daten wird das AIS systematisch trainiert, so dass es die Anzeigen immer besser aussteuert (Fesefeldt 2018).

Eng hiermit zusammen hängt das Recruiting, in dem ein großes Feld für den Einsatz autonomer Informationssysteme zu sehen ist. So wird zuweilen von „Robot Recruiting" gesprochen, wenn die Suche nach relevanten Personalinformationen, das Matching von Stelle und Stellensuchenden, die Anwerbung oder auch eine erste Diagnostik der Fähigkeiten durch das AIS und nicht mehr durch den Menschen erfolgen (Fesefeldt 2018). AIS informieren potenzielle Interessente nicht nur über Details der vakanten Stellen und das Unternehmen. Sie führen mit den Interessenten Dialoge, die neben berufsbezogenen Fragen auch Sprache und Mimik erfassen, um selbstständig eine erste Vorauswahl zu treffen. Die Ergebnisse erhält der menschliche Personaler, der auf dieser Basis weitere gezielte Fragen stellen kann. Mit einem derartigen Vorgehen hat das Unternehmen Unilever beispielsweise von 2016 bis 2017 in mehr als 50 Ländern in 15 Sprachen ca. 250.000 Hochschulabsolventen getestet und davon 800 eingestellt. Die Effekte waren immens: Verkürzung der Zeit von Ansprache bis Einstellung von 4 Monaten auf 4 Wochen; eingesparte Kosten in Höhe von ca. 1 Mio. $€$, Verdopplung der Zahl der Bewerbungen und Halbierung der Zahl der Abbrüche. Ähnlich funktioniert die Software „Talk’n Job“, die im Einzelhandel in Deutschland eingesetzt werden soll (Hoffmeyer 2020). Per Chat und Sprachsteuerung werden interessierten Bewerbern durch das AIS Fragen gestellt und deren Antworten analysiert, so dass der Personaler eine gute Basis für die nächsten Auswahlschritte erhält. Deutlich wird auch hier: vormals menschlich durchgeführte oder gesteuerte Analyse- und Schlussfolgerungsprozesse werden durch AIS ersetzt, um Entscheidungen zu treffen oder Entscheidungsvorlagen für den Menschen zu generieren.

Wie schon angesprochen, kann AIS-basierte Personalauswahl zu besseren, schlechteren oder ähnlich guten oder schlechten Ergebnissen gelangen wie die menschliche. Wird, wie bei Amazon, ein voreingenommener Datensatz zum Trai- 
ning des AIS verwendet, so sind selbstverständlich auch die mit AIS generierten Entscheidungen voreingenommen. Ob Entscheidungen des AIS tatsächlich der menschlichen Entscheidung überlegen sind und den Menschen bei der Personalauswahl ersetzen werden, lässt sich gegenwärtig noch nicht abschließend beurteilen. Die auch bei menschlichen Auswahlentscheidungen thematisierte fehlende Diversität kann durch AIS verschärft oder auch entspannt werden. Vielleicht liegt hierin auch die Chance, dass Personaler durch AIS lernen, indem sie neutral nachvollziehbare Entscheidungen treffen, die allen gleich gut qualifizierten Kandidaten die gleichen Chancen ermöglichen (Chamorro-Premuzic et al. 2019).

Auch in der Personaleinsatzplanung oder in der Projektplanung können AIS zum Einsatz kommen, indem sie die Projekt- und Schichtplanung selbstständig übernehmen (Klausch 2019), die Analyse sowie den Einsatz der für ein Projekt vorgesehenen Ressourcen autonom durchführen oder auch Teams problem- und aufgabenorientiert konfigurieren (Sattelberger 2016; Picot 2016). Erste Beispiele aus Unternehmen, in denen die Konfiguration von Teams ohne menschlichen Eingriff durch AIS erfolgt, zeigen gerade hier ein großes Einsatzfeld. Basis sind Daten zu Kompetenzen, Ressourcen, Erfahrungen, Verfügbarkeiten etc., bei deren Verdichtung, Analyse und Auswertung AIS schneller und effizienter zu Entscheidungen kommen können. Unterstützen lässt sich schließlich auch die Personalentwicklung (Klausch 2019). AIS können Kompetenzanalysen durchführen, individuelle Lernpfade erstellen, problemorientiert und individuelle Lerninhalte konfigurieren und letztlich Angebot und Nachfrage nach Lerninhalten matchen. Chatbots können Fragen beantworten und Kontrollfunktionen übernehmen. Auch Tutorials, Schulungen oder problemorientierte Lösungshilfen lassen sich durch AIS realisieren.

Schließlich rückt ein weiteres Feld zunehmend in die Diskussion: die Möglichkeit der Leistungserfassung durch AIS. Je mehr Daten über die Arbeits- und Leistungsprozesse der Mitarbeiter erfasst werden, desto besser können AIS individuelle Leistungen bewerten, Performance messen, Profiling durchführen sowie auf der Basis all dieser Daten und Informationen Schlussfolgerungen mit z. T. weitreichenden Konsequenzen für die Betroffenen ziehen. Hierzu zählen z. B. Konsequenzen für die Gewährung personenbezogener Anreize und Boni, Bestimmung individueller Lern- und Qualifikationspfade, Zuordnung von Aufgaben oder auch die Planung der jeweiligen Karrierepfade. So setzt beispielsweise das Unternehmen Bridgewater Associates ein Tool ein, das das von den Mitarbeitern gesendete positive und kritische Feedback selbstständig analysiert und die erhaltenen Ergebnisse direkt mit dem Vergütungssystem verknüpft (Scheelen 2019). Vormals vom Menschen durchgeführte Analyseprozesse werden an das Tool delegiert.

Je mehr und je bessere Daten zur Verfügung stehen und je kontextorientierter AIS trainiert sind, desto qualitativ besser und aussagekräftiger werden die Ergebnisse. AIS führen zu einer tatsächlichen oder wahrgenommenen höheren Transparenz im Bereich der Leistungserfassung und Leistungsbewertung. Besteht die Gefahr, dass hiermit Nachteile für den Betroffenen verbunden sind, wirkt sich diese u. U. kontraproduktiv auf Motivation und Leistungsbereitschaft aus. Allerdings ist höhere Transparenz nicht nur negativ zu beurteilen (Gierlich et al. 2020). Sie kann auch einen Beitrag zum vertrauensvolleren Umgang (Fiedler und Haruvy 2016) und zum Schutz der Mitarbeiter leisten, wenn diese auf Grund der erfassten Daten beispielsweise vor 
Bore-Out aber auch Selbstausbeutung und Burn-Out bewahrt werden können. Möglicherweise ziehen AIS hier sogar bessere und fundiertere Schlussfolgerungen, da sie derartige Verhaltensweisen neutraler als der Mensch beurteilen.

Im Ergebnis stellen sich hier ähnliche grundlegende Fragen wie zuvor im Bereich der Führung: welche HR-Aufgaben und -Entscheidungen übernimmt das AIS autonom, welche erfolgen gemeinsam und welche verbleiben rein beim Menschen? Im Vergleich zu den Überlegungen zur Unternehmensführung entwickelt sich die Arbeitsteilung hier jedoch etwas anders, da die zugrundeliegenden Schlussfolgerungsund Entscheidungsprozesse in vielen Bereichen einen höheren Grad an Strukturiertheit und geringer Veränderlichkeit haben und damit trainierbar sind. In Konsequenz ist davon auszugehen, dass im HR-Bereich - so wie wir ihn bisher kennen - zunehmend Analyse-, Schlussfolgerungs- und Entscheidungsprozesse durch AIS durchgeführt werden. Gelingt es zudem, die zugrundeliegenden Daten zu verknüpfen - indem z.B. die Daten aus der Teamkonfiguration mit den Daten aus der Personalentwicklung verbunden werden - lassen sich womöglich auch übergreifende situationsspezifische Schlussfolgerungen ziehen, wodurch sich die Einsatzfelder der AIS weiterentwickeln. Lassen sich nun mehr und mehr Prozesse auf AIS delegieren, entsteht die Chance, HR neu auszurichten und in enger Zusammenarbeit mit dem Management strategische Themen wie Talent Management, Kompetenzentwicklung oder People Strategy stärker in den Vordergrund zu stellen. Von den Mitarbeitern im HR-Bereich sind dann veränderte und angepasste Kompetenzen gefragt. Diese betreffen v. a. strategisches Denken und Beurteilungskompetenzen, Kreativität sowie Denken in multi-perspektivischen Zusammenhängen (vgl. Abschn. 4).

\subsection{Konfiguration und Koordination von Prozessen und Strukturen - AIS als Architekten zukünftiger Organisationen?}

Als drittes beispielhaftes Einsatzfeld sei die Koordination und Steuerung von Prozessen und Strukturen in und zwischen Organisationen durch AIS angeführt. Auch hier lassen sich vielfältige Einsatzfelder erkennen. So unterstützen z. B. assistierende Mess- und Prüfsysteme im Triebwerksbau Mitarbeiter in der Montage, indem sie fehlerhaft montierte Einzelteile sicher erkennen, eine sofortige Fehlerkorrektur anbieten und diese Prozesse durch direktes Feedback verbessern (Haase et al. 2015). Autonome Schreibprogramme erstellen journalistische Beiträge, die sich für den Leser oft nicht mehr von menschlich geschriebenen unterscheiden. Audio-zu-Textbzw. Video-zu-Text-Converter, Übersetzungsdienste oder Chatbots übernehmen eigenständig zahlreiche Kommunikationstätigkeiten in korrespondierenden Berufen, die bisher von Sekretariats- und Assistenzkräften, Fremdsprachenkorrespondenten, wie auch Mitarbeitern in Marketing und Öffentlichkeitsarbeit (Weber 2019) durchgeführt wurden. Legal Chatbots sind immer besser in der Lage, Sachverhalte zu erfassen, die Rechtslage selbstständig zu beurteilen, erforderliche Schritte zu initiieren und Handlungsempfehlungen zu geben (Legal Tech 2020). In Folge lassen sich juristische Prozesse, wie beispielsweise rechtliche Analysen und Beurteilungen auf der Basis der Anwendung rechtlicher Regelwerke an das AIS delegieren, Legal Chatbots in Wissensbasen implementieren oder die Gestaltung von Verträgen bzw. die notarielle Beurteilung durch AIS durchführen (Wilkens und Falk 2019). Autonom 
agierende Roboter und Transportmittel, selbstständig urteilende Mess- und Prüfsysteme wie auch autonome Sichtungs- und Sortierhilfen übernehmen mehr und mehr vormals manuell durchgeführte Produktionsschritte (Gatzke et al. 2018). Erschienen ist mittlerweile auch die erste durch AIS generierte wissenschaftliche Metastudie zu Lithium-Ionen Batterien, bei der der Mensch ausschließlich Editierungsaufgaben übernommen hat (Writer 2019). Deutlich wird: AIS führen in zahlreichen Einsatzfeldern auf der Basis von früher ausschließlich vom Menschen getätigte Analyse-, Entscheidungs- und Schlussfolgerungsprozesse eigenständig durch.

Letztlich ist die Steuerung von Maschinen, Prozessen und Personen durch AIS in Echtzeit denkbar, wie sie schon jetzt in Industrie 4.0-Szenarien thematisiert wird (Obermaier 2019). Die Konsequenz sind voll automatisierte Fabriken und Produktionshallen, die im Extremfall weitgehend ohne menschlichen Eingriff funktionieren. Ein Beispiel hierfür war die Adidas Speedfactory, in der die Schuh-Produktion weitgehend autonom erfolgte (Busse 2017). Auch Crowd-Working-Plattformen lassen sich hier anführen (Benner 2014; Vogl 2018), denn letztlich erfolgen sowohl Zuordnung und Konfiguration von Tätigkeiten auf Crowd-Working- oder CrowdSourcing-Plattformen wie auch Steuerung und Kontrolle weitgehend durch AIS (Ihl et al. 2018). Gerade hier entsteht zusätzliche Komplexität, da Abwägungsprozesse erforderlich werden, um zu viele Veränderungen und Anpassungen und dadurch resultierende Unruhe im Team oder in der Organisation zu vermeiden.

Die Fähigkeit der Echtzeit-Steuerung von Prozessen in Verbindung mit der Fähigkeit, Teams autonom zu koordinieren versetzen AIS zukünftig in die Lage, nicht nur einzelne Aufgaben wie beim Crowd-Working, sondern ganzheitliche Wertschöpfungsprozesse anders zu koordinieren und zu steuern. Denkbar ist hier ein Szenario, in dem der Wertschöpfungsprozess in modulare Teilprozesse gegliedert wird (Picot et al. 2020b; Gregory et al. 2020; Rai et al. 2019), deren Koordination und Steuerung ausschließlich durch AIS erfolgt. Das AIS entscheidet dabei selbstständig auf der Basis von Daten über die Eigenschaften der Teilprozesse einerseits sowie die jeweiligen Kompetenzen andererseits, wer (interne Abteilung, Freelancer, Kooperationspartner, externes Unternehmen, Crowd-Worker etc.) den jeweiligen Teilprozess durchführt. Je modularer und abgegrenzter sich die Teilprozesse abbilden lassen, desto eher lassen sie sich denjenigen Akteuren, die genau auf die jeweils geforderten Tätigkeiten spezialisiert sind, zuordnen. Ändert sich die zugrundeliegende Datenlage, passen AIS die organisatorische Konfiguration an. Im Ergebnis entsteht eine durch AIS konfigurierte Form der flexiblen unternehmensübergreifenden Arbeitsteilung. Voraussetzung sind auf der technischen Ebene Standards; auf der organisatorischen Ebene Kooperationsbeziehungen quasi als Rahmen für die flexible Koordination (Kilian et al. 1994).

$\mathrm{Ob}$ sich derartige Strukturen weiter durchsetzen werden, ist derzeit offen. Falls ja, könnte dieser Trend zu einer zunehmenden Hyperspezialisierung führen. Je spezialisierter die Akteure sind, desto höher ist die Chance, vom AIS berücksichtigt zu werden. Der ohnehin erkennbare Trend zum Outsourcing und damit verbundener Trend zur Auflösung existierender Unternehmensgrenzen (Picot et al. 2020b) setzt sich fort, wenn AIS die Koordination von Aufgaben übernehmen. Basierend auf einem Prozess zur Aufgabenkonfiguration, der mit jeder vollzogenen Konfiguration lernt und besser wird, übernimmt das AIS hier klassische Koordinierungs- 
Abb. 1 Einfluss von AIS auf Aufgaben in Organisationen

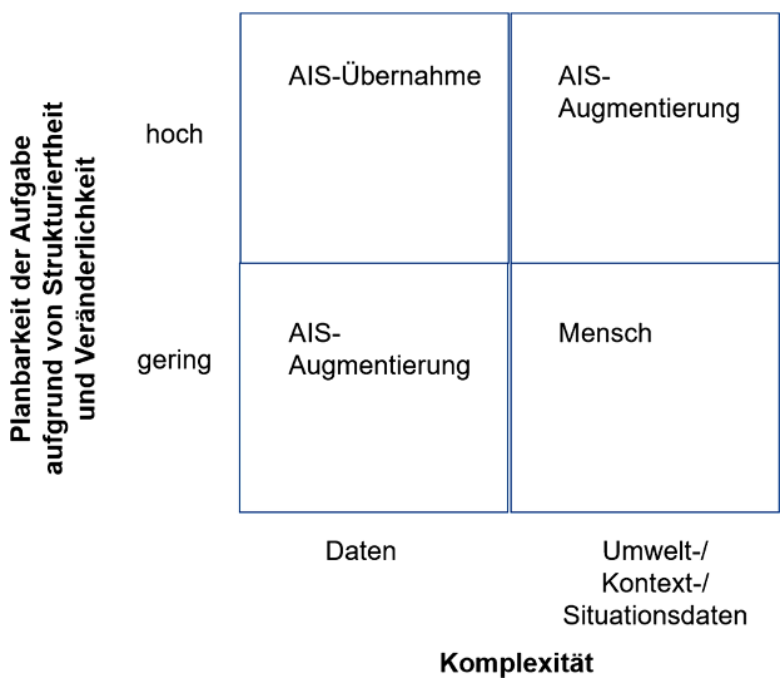

und Steuerungsprozesse. Langfristig vorstellbar ist hier durchaus ein Szenario, dass das Management sich auf die Entwicklung innovativer Ideen konzentriert und die organisatorische Umsetzung dieser Ideen dann das AIS übernimmt. Aufgabe des Managements ist es dann wiederum, den gesamten Prozess im Blick zu haben. Auch hier lässt sich erkennen: das AIS übernimmt Such-, Auswahl-, Steuerungsund Kontrollprozesse, die vormals durch Menschen durchgeführt wurden. Dies hat nicht nur strukturelle Konsequenzen, indem noch flexiblere Strukturen entstehen. Es hat v. a. auch Auswirkungen auf die von diesen Entscheidungen betroffenen Personen, die sich auf flexible Koordinationsmechanismen einstellen müssen. Hierfür sind wiederum veränderte Kompetenzen erforderlich (vgl. Abschn. 4).

\subsection{Zwischenfazit: Neue Arbeitswelt durch und mit AIS?}

Zusammenfassend lässt sich an dieser Stelle erkennen: prinzipiell können AIS derzeit eigenständig Aufgaben und Entscheidungen übernehmen. Allerdings handelt es sich um einen eher kleinen Teil von Aufgaben, nämlich die aufgrund hoher Strukturiertheit und geringer Veränderlichkeit gut planbaren Aufgaben, die sich durch eine hohe Datenkomplexität auszeichnen (vgl. Abb. 1 links oben).

Dennoch ist festzustellen, dass AIS in Form einer Augmentierung schon in eine Reihe von Aufgabengebieten zusätzlich eingedrungen sind, die entweder gut planbar oder durch Datenkomplexität charakterisiert sind (vgl. Abb. 1). Je mehr sie zum Einsatz kommen, desto besser können sie lernen und trainiert werden und desto mehr sind sie in der Lage, noch bessere Schlussfolgerungen zu ziehen. Im Ergebnis verändern sich so Arbeitswelt und Rollen der einzelnen Akteure und stellen so neue Anforderungen an die notwendigen Kompetenzen der Mitarbeiter und Manager.

Veränderung der Arbeitsteilung zwischen Mensch und autonomen Informationssystem. AIS können prinzipiell Führungs- oder operative Ausführungsaufgaben 
übernehmen und werden einen festen Bestandteil in der zukünftigen Arbeitswelt darstellen. Die Arbeitsteilung zwischen Mensch und AIS wird sich in bestimmten unternehmerischen Handlungskontexten verschieben.

Zunächst übernimmt der Mensch eine veränderte Rolle in dem sich verändernden Arbeitssystem. Er delegiert Entscheidungs-, Schlussfolgerungs- und Analyseprozesse auf das AIS; er trifft Entscheidungen auf der Basis von Schlussfolgerungen und vorbereiteten Entscheidungen durch das AIS; er kontrolliert die Entscheidungsprozesse des AIS oder wird von den Entscheidungsprozessen des AIS gesteuert und kontrolliert; er trainiert AIS durch Daten und Rückkopplungen über die gezogenen Schlussfolgerungen und lernt aus ihren Schlussfolgerungen, indem er neues Wissen zur Lösung konkreter Probleme oder strategischer Herausforderungen aufbaut.

Sind Aufgaben gut strukturiert und gering veränderlich und zeichnen sich zudem durch Datenkomplexität aus, ersetzen AIS vormals durch den Menschen durchgeführte Analyse-, Schlussfolgerungs- und Entscheidungsprozesse. Mit abnehmender Planbarkeit werden AIS in veränderten Formen der Ko-Existenz zusammenarbeiten, indem sie die jeweiligen menschlichen Stärken in spezifischen Domänen ergänzen. Schließlich wird es Aufgaben und Entscheidungen geben, bei denen die Vorhersehbarkeit auf Grund von geringer Strukturiertheit und hoher Veränderlichkeit sowie von Umwelt- und Kontextkomplexität erschwert ist. Hierzu gehören beispielsweise nachhaltige, ethische oder soziale Entscheidungen sowie Zusammenhangserkenntnisse, die auf langjährigem Erfahrungswissen sowie einer tiefen Situationskenntnis basieren. Sie verbleiben voraussichtlich erstmal beim Menschen. Bedingt durch ihre Lernfähigkeit und die Möglichkeit zum Training dieser Systeme, verbessern sich AIS jedoch, so dass zukünftig zu erwarten ist, dass AIS mehr und mehr auch dieser Prozesse, zumindest augmentierend, übernehmen werden können.

Veränderung der Organisations- und Monitoringstrukturen. Übernehmen AIS zudem die Koordination erforderlicher Wertschöpfungsprozesse, entstehen flexible organisatorische Strukturen, bei denen die Einbindung externer Wertschöpfungspartner algorithmenbasiert durch AIS erfolgt. Die klassische organisatorische Aufgabenteilung nach Funktionen, Produkten, Prozessen oder auch Regionen verliert nochmals mehr an Relevanz; die Tendenz zu vernetzten Strukturen wie Industrie 4.0 sowie der damit eng zusammenhängenden Forderung nach einer Losgröße 1 wird nochmals verstärkt. An die Stelle linearer Prozesse als Gestaltungskriterium treten Vernetzung sowie aufgaben- oder problemorientierte Konfigurationen, die beispielsweise durch AIS flexibel angepasst werden.

Gleichzeitig entstehen neuartige Monitoringstrukturen. AIS binden menschliche Arbeit nicht nur in die Arbeitsprozesse ein; sie kontrollieren sie auch. So führt die oben thematisierte Leistungserfassung durch AIS zu neuartigen Formen der Kontrolle. Der Mitarbeiter nimmt zwar erweiterte Handlungsspielräume wahr, da er beispielsweise dezentral eingebunden ist in den Prozess. Gleichzeitig werden diese beschränkt durch digitale Leistungserfassung und höhere Transparenz. Beispiel ist der Crowdworker, der zwar Autonomie bei der Übernahme und Gestaltung seiner täglichen Arbeitsprozesse hat, gleichzeitig jedoch durch die Algorithmen der Plattform gesteuert und kontrolliert wird. Hierdurch ergeben sich ganz neuartige Anforderungen an Mitbestimmung und Datenschutz. So werden in den gegenwärti- 
gen Regelungen zur Mitbestimmung weder derartige flexible Strukturen noch deren Koordination durch AIS berücksichtigt. Dass die Leistungserfassung wiederum nur mit Einwilligung der Betroffenen erfolgen darf, zeigt letztlich auch, dass die Grenzen der Einsatzfähigkeit derartiger AIS nicht nur in den technischen Möglichkeiten bestehen. Zudem kommt der große Bereich potenzieller Verzerrungen im Algorithmus, der zu fragwürdigen Vorhersagen und Entscheidungen beiträgt. Hier stellen sich wie besprochen völlig neue Anforderungen an die Regulierung und das Monitoring der Erklärbarkeit und Nachvollziehbarkeit des AIS.

Veränderung von Anforderungen und Herausbildung neuartiger Tätigkeits- und Kompetenzprofile. Insbesondere durch die Substitutionseffekte führen AIS zu einer sehr viel geringeren Nachfrage bestimmter beruflicher Anforderungen und erforderlicher Qualifikationen. Häufig wird in diesem Fall auch von De-qualifizierung und Verlust von Erklärungskraft der Organisation gesprochen. Gleichzeitig entstehen neuartige Tätigkeiten im Bereich von Interaktion, Verantwortung, Steuerung/ Kontrolle, Identifikation von Bereichen, die sich nicht für AIS eignen und gegenseitigem Lernen. Vor dem Hintergrund von Abb. 1 handelt es sich insbesondere um schlecht planbare Aufgaben mit Umwelt- und Kontextkomplexität. Hier dürfte der Einsatz von AIS ohne entsprechende überwachende und eingreifende menschliche Expertise problematische Konsequenzen haben, da AIS derzeit noch nicht gut mit unerwarteten Ereignissen im Kontext umgehen. Zudem bilden sich neuartige Kompetenzprofile heraus. Diese reichen vom Architekten und Trainer der AIS, Innovatoren, Problemlöser über die Weiterentwicklung und Pflege von AIS bis hin zum Agieren als direkte oder indirekte Feuerwehr, wenn AIS ausfallen oder schlecht entscheiden. Zudem ergeben sich durch die AIS neue Aufgaben, Verantwortlichkeiten und Rollenidentitäten. In Folge stellt sich die Frage, welche Kompetenzen die durch den Einsatz von AIS direkt oder indirekt tangierten Menschen in ihren Rollen als Entwickler/Programmierer, Anwender und als Trainer zukünftig benötigen und welche Anforderungen sich an die Entwicklung dieser Kompetenzen stellen.

\section{Zukünftige Herausforderungen: Kompetenzentwicklung als strategische Aufgabe}

Ausgehend von den skizzierten AIS-Entwicklungen stellt Kompetenzentwicklung eine strategische Aufgabe für viele Organisationen dar. Physische und operative Tätigkeiten als auch kognitive Aufgaben, die planbar sind, werden weiter in ihrer Bedeutung sinken, während nicht vorhersagbare Aufgaben, die Kommunikationsund Verhandlungsgeschick, Kreativität und kritisches Denken, aber auch die Fähigkeit, mit AIS zu interagieren und sie informationstechnisch einordnen zu können, in ihrer Bedeutung steigen werden. Entwickler und Programmierer von AIS benötigen Spezialwissen insbesondere in Künstlicher Intelligenz, Deep Learning und Machine Learning. Menschen, die AIS trainieren, müssen in der Lage sein, die relevanten Datenbestände im Hinblick auf ihre Nutzung durch AIS zu bewerten und zugänglich zu machen, Fälle klassifiziert bereitzustellen und vom AIS durchgeführte Schlussfolgerungen für die jeweilige Situation zu überprüfen. All die hier zukünftig relevanten 
Abb. 2 Kompetenzentwicklung für eine veränderte Arbeitsteilung zwischen Mensch und AIS

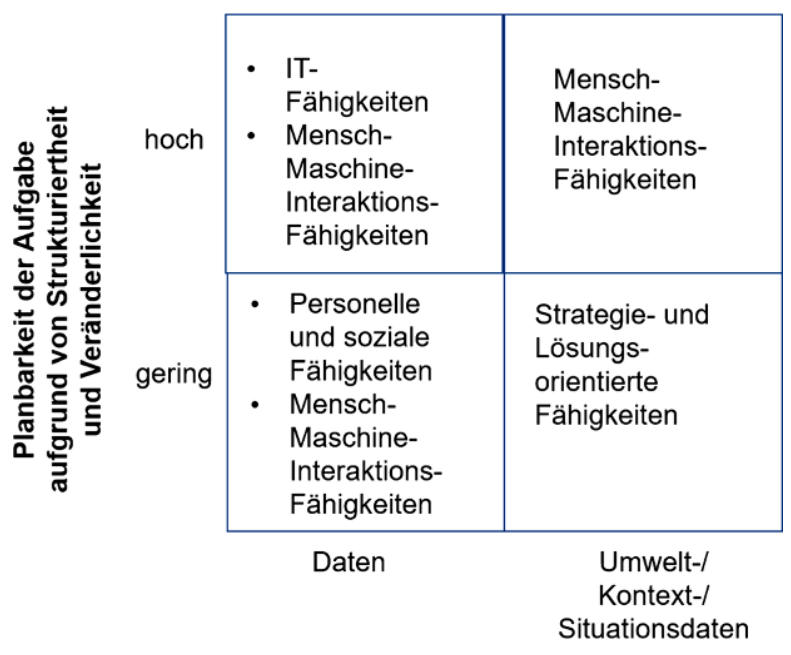

Komplexität

Fähigkeiten unterteilen wir im Folgenden in IT-Fähigkeiten, Mensch-Maschine-Interaktions-Fähigkeiten, Personelle und Soziale Kompetenzen als auch Strategie- und Lösungsorientierte Fähigkeiten (MÜNCHNER KREIS 2020). Je nach zugrundeliegender Rolle von Mensch und AIS (vgl. Abb. 1) sind sie unterschiedlich relevant (vgl. Abb. 2).

\subsection{IT-Fähigkeiten}

Insbesondere zur Entwicklung von AIS, die eigenständig Aufgaben in der Organisation übernehmen, sind IT-Fähigkeiten notwendig. Auch wenn AIS eigenständig lernen können, müssen sie regelmäßig gewartet und weiterentwickelt werden. Dies erfordert insbesondere IT-Kompetenzen auf mehreren Ebenen. Zunächst sind grundlegende digitale Kenntnisse oder Basis-IT-, KI- und Programmierkenntnisse erforderlich, um das zugrundeliegende Zusammenspiel aus Software/Algorithmen und Hardware zu verstehen und beides ganzheitlich als System kontinuierlich verbessern und weiterentwickeln zu können. Die Basis von AIS ist eine Vielzahl unterschiedlicher, strukturierter oder weniger strukturierter Daten, für deren Erfassung, Einordnung und Analyse grundlegende Big-Data-Kompetenzen wie Data Science oder Data Analytics erforderlich sind (Picot und Neuburger 2020). Zur vertieften Analyse und insbesondere zum Training von AIS sind schließlich tiefergehende Kenntnisse in den verschiedenen maschinellen Lernverfahren wie auch das Verständnis, in welchen Fällen welches maschinelle Lernverfahren zum Einsatz kommen kann, erforderlich. $\mathrm{Zu}$ berücksichtigen ist auch, dass mit AIS sich derzeit ausschließlich Daten in Entscheidungen einbeziehen lassen, die in digitaler Form vorliegen, so dass die Schaffung einer Infrastruktur, die das Lernen von großen Datenmengen erlaubt, ebenfalls zu diesem Bereich an notwendigen Fähigkeiten gehört. Letztlich sind selbstverständlich auch IT-Fähigkeiten nötig, die das Design von digitalen Plattformen erlauben, die möglichst unverzerrte Entscheidungen ermöglichen. 


\subsection{Mensch-Maschine-Interaktions-Fähigkeiten}

AIS stellen ein vergleichsweise neues Element in der Arbeitswelt dar, mit denen Menschen in unterschiedlicher Weise zusammenarbeiten. Sie entwickeln und programmieren AIS, sie wenden AIS an und sie trainieren sie. Für diese Zusammenarbeit müssen Menschen in Mensch-Maschine-Interaktionsfähigkeiten ausgebildet und vorbereitet sein und die Entscheidung des AIS nachvollziehbar sein. Dies beginnt mit einem grundlegenden Verständnis der Art, des Umfangs und der Legitimität der Mensch-Maschine-Interaktionen in ihren Unterschieden und der sich damit verändernden Rolle des Menschen in der Arbeit. Nur so lassen sich durch das AIS vorgenommene diskriminierende und falsche Entscheidungen identifizieren und fehlerhaft eingeschlagene Pfade verlassen. Insofern liegt hierin nicht nur eine hohe Verantwortung bei den Programmierern, sondern auch bei den Anwendern der AIS. Sie müssen die Zusammenhänge verstehen und vor dem Hintergrund des jeweiligen Kontextes bewerten. Dazu kommen Fähigkeiten zur direkten Anwendung bzw. Zusammenarbeit mit dem AIS - sei es den Einsatz eines Chatbots, die Zusammenarbeit mit einem Roboter im Produktionsprozess oder die Nutzung einer KI-unterstützten Softwareanwendung im Rahmen des HR-Managements. Dabei stellt sich die Frage, ob der Mensch Fähigkeiten in Richtung Umgang mit der AIS-Entscheidung oder in Richtung Prüfung der AIS-Entscheidung entwickeln muss, um seine berufliche Identität zu finden. Eine weitere wichtige Mensch-Maschine-Interaktions-Fähigkeit besteht schließlich darin, AIS zu trainieren. Je nachdem welche maschinellen Bausteine und Lernverfahren zum Einsatz gelangen, erfordert dies vertiefte Kenntnisse nicht nur in Data Science, sondern v. a. auch in Software-Tools und Prozessoren zum Trainieren der Algorithmen.

\subsection{Personelle und Soziale Fähigkeiten}

Das Agieren mit AIS erfordert zunächst Beurteilungsfähigkeit, um Entscheidungen von AIS reflektieren zu können, sowie die Fähigkeit, durch die Schlussfolgerungen des AIS zu profitieren und zu lernen. Durch AIS werden sich flexiblere Prozesse und Strukturen herausbilden. Das Arbeiten in derartigen dezentralen Strukturen erfordert zum einen Fähigkeiten wie Eigen- und Selbstverantwortung und Selbstorganisation; zum anderen soziale Kompetenzen wie Kommunikation, Konfliktfähigkeit und Teamarbeit. Auch verlangt es von Anbietern derartiger Systeme, dass sie diese Fähigkeiten bei der Gestaltung von Arbeitsprozessen mitdenken. So zeigt sich, dass die mit AIS geführten Microtask-Crowdworker stark auf die affektive soziale Unterstützung anderer menschlicher Crowdworker zurückgreifen, was sich wiederum positiv auf ihre Identifikation mit der Gruppe der Crowdworker, den in der Arbeit gesehenen Sinn und damit ihr Engagement auswirkt (Ihl et al. 2020).

\subsection{Strategie- und Lösungsorientierte Fähigkeiten}

Auch zukünftig wird es Aufgaben und Entscheidungen geben, die speziell und alleine von Menschen durchgeführt werden (vgl. Abb. 1). Hierzu gehören v.a. die schlecht planbaren Aufgaben, die tiefe Situationskenntnisse und damit kausales 
Verständnis erfordern, wie z.B. die Entwicklung von ad-hoc-Lösungen, wenn Roboter zu einem Produktionsstopp führen, die Weiterentwicklung von nachhaltigen Geschäftsmodellen, Produkten oder Services oder auch das Reagieren auf ad-hoc wegbrechende Märkte im Zuge von Umweltkatastrophen oder Krisen.

Dieses kausale menschliche Verständnis entwickelt sich nur durch langjährige Erfahrung in einer Domäne und den Drang zur ständigen Weiterentwicklung (Fiedler 2004). Dabei unterscheidet sich menschliche Problemlösung von der in Kap. 2 dargestellten AIS-Herangehensweise. Polanyi (1957) beschreibt den menschlichen Problemlösungsprozess so, dass die Intuition zunächst verborgene Ressourcen zur Problemlösung lokalisiert, eine Idee respektive Problemstellung liefert und die Imagination in ihrem Streben nach Problemlösung ausrichtet. Nach Polanyi (1966) ist menschliche Expertise dann am Werk, wenn die sogenannte implizite Triade intakt ist, d.h. wenn der Mensch seine erworbenen proximalen Terme (sozusagen die Bausteine seines Wissens) auf den sogenannten distalen Term (die Frage, die es zu lösen gilt) mit dem soeben dargestellten Problemlösungsprozess anwendet. So kann der erfolgreiche Manager aufgrund seiner erworbenen proximalen Terme (z.B. Ausbildung, Erfahrung, Branchenkenntnis, Kultur usw.) überdurchschnittlich schnell erkennen, um welche Art von Problem es sich handelt und entsprechende Maßnahmen zur Lösung einleiten (Fiedler 2004). Die menschliche Expertise ist dabei meist auch körperlich verankert, da sie nicht nur über Lesen von Informationen, sondern auch über das begleitende Training des Auges, des Ohrs und der Berührung trainiert wird (Polanyi und Prosch 1975; Fiedler 2004). An dieser Stelle wird auch klar, dass der Mensch versucht basierend auf seinen erworbenen proximalen Termen eine individuelle kausale Interpretation der distalen Terme zu finden, was ihn von einer AIS-Herangehensweise unterscheidet, das Vorhersagen aufgrund von Zusammenhängen trifft. Ein Arzt sieht einen Patienten an und versucht (basierend auf seinen proximalen Termen) eine individuelle Diagnose hinsichtlich des zukünftigen Behandlungsbedarfs dieses spezifischen Patienten zu treffen. Das AIS trifft diese Behandlungsempfehlung basierend auf dem in Kap. 2 dargestellten Trainingsprozess über die Kategorisierung des Patienten, ohne die tatsächliche Ansicht des Individuums, jedoch natürlich unter zu Hilfenahme von Daten des Patienten.

All diese Kompetenzfelder sind nicht neu; gewinnen aber im Zuge des Zusammenwirkens zwischen Mensch und AIS eine neue Relevanz und müssen somit in ein für die Zukunft ausgerichtetes Kompetenzmanagement einfließen. Daneben geht es aber auch um die Erhaltung bestimmter Kompetenzen. Denn je mehr bestimmte Arbeitsprozesse oder Tätigkeiten durch AIS übernommen werden, desto mehr verliert der Mensch die Fähigkeit, diese Prozesse noch selbst zu tätigen, da ihm die Übung und mit der Zeit auch das notwendige Erfahrungswissen dazu fehlen, bzw. bei Neueinstellung für diese Aufgaben, nie vorhanden war. Dieses auch als „Ironie der Automatisierung“ bezeichnete Phänomen (Bainbridge 1983) mag für bestimmte Prozesse unproblematisch sein, insbesondere, wenn sie vom AIS gänzlich fehlerfrei bzw. besser als der Mensch übernommen werden. In den Fällen, in denen jedoch Erklärungsfähigkeit und schnelle Einflussnahme des Menschen wichtig sind, sollten diese Kompetenzen im Unternehmen gehalten werden. Dies betrifft v. a. auch die Frage, wie stark der Mensch die jeweiligen vom AIS durchgeführten Grundprobleme kennen muss, um im Notfall einzugreifen oder entstehende Sonderprobleme lösen 
zu können. Beispiel ist das menschliche Einspringen für Kundenanfragen, wenn der Chatbot plötzlich ausfällt oder die Einflussnahme des Piloten, wenn der Autopilot nicht schnell oder gut genug reagiert.

Die gezielte Entwicklung derartiger Kompetenzen im Arbeitskontext stellt hohe Anforderungen an Organisationen. Auf organisatorischer Ebene gilt es zu entscheiden, wie Ressourcen investiert werden und welche Organisationsform sich hierfür eignet. Hierzu gehört auch, die sich verändernde Aufgaben- und Entscheidungsteilung zwischen Mensch und AIS strukturell umzusetzen und die skizzierten Kompetenzen zu fördern. Aufgabe der Führung ist es, Ressourcen für die Kompetenzentwicklung zur Verfügung zu stellen und eine entsprechende lernmotivierende Umgebung zu schaffen. Anzupassen sind gleichzeitig die Motivations- und Anreizsysteme. Diese sind an sich ändernde Entscheidungs- und Aufgabenstrukturen anzupassen; zum anderen sind das Sammeln und Reflektieren von Erfahrungen, Kompetenzentwicklung und Lernen gezielt zu fördern. Eine besondere Rolle kommt hier v. a. dem Human Ressourcen-Management zu. Durch die Substitution bestimmter Prozesse durch AIS entstehen Freiräume, die sich für eine vorausschauende Personalplanung im Dialog mit Fachverantwortlichen und Unternehmensführung nutzen lassen und die Mitarbeiter zur Findung einer neuen Rollenidentität auffordern.

\section{Ausblick}

AIS dringen in die Arbeitswelt ein und verändern zunehmend die Arbeitsteilung mit Menschen. Neue Elemente sind dabei der Übergang der Entscheidung und des Vorhersagens vom Menschen auf das AIS und die selbstständige Weiterentwicklung des Systems auf der Basis von Daten, Training und Feedback. Dadurch erhält die schon seit jeher geführte Diskussion der Arbeitsteilung zwischen Mensch und Maschine eine neue Dimension. Es geht um die Abgabe bzw. die Teilung von Entscheidungsgewalt und Verantwortung in einer zukünftigen Arbeitswelt. Dabei ist das AIS nicht immer als Wettbewerber für den Menschen zu sehen, der dem Menschen zukünftig sämtliche Tätigkeiten und Verantwortungsbereiche abnimmt. Vielmehr lassen sich oft die jeweiligen Stärken des Menschen und des AIS integrieren und in kooperativer Koexistenz augmentiert zum Einsatz bringen. So liegen die Stärken menschlicher Akteure eher in der Bewältigung von Prozessen, die insbesondere auf Grund der geringen Strukturiertheit und hohen Veränderlichkeit nicht vorhersehbar sind und sich durch hohe Umwelt- und Kontextkomplexität auszeichnen. Dagegen haben AIS eher ihre Stärken bei Prozessen, die planbar sind und bei denen die Beherrschung einer hohen Daten- und Analysekomplexität erforderlich ist. Die Herangehensweise, wie Mensch und AIS ihre Aufgaben und Prozesse bewältigen, ist dabei selbstverständlich unterschiedlich. Während Menschen über Intuition und Imagination Probleme lösen und nach kausalen Zusammenhängen suchen, macht das AIS Vorhersagen ohne derartiges Verständnis. Dementsprechend bestehen Anforderungen an die Nachvollziehbarkeit und Erklärbarkeit entsprechender Entscheidungen, um frühzeitig Verzerrungen entgegenzuwirken. Gleichzeitig bietet dieses Vorgehen und die Anforderungen an ein kontinuierliches Audit des AIS aber auch die Mög- 
lichkeit, Verzerrungen im menschlichen Entscheiden aufzudecken und Menschen dazu zu bringen, sich mit ihren proximalen Termen auseinander zu setzen.

Im Ergebnis werden AIS gewisse Prozesse und Entscheidungen gänzlich übernehmen; andere führen sie in kooperativer Ko-Existenz mit menschlichen Akteuren aus; andere wiederum verbleiben auch zukünftig beim Menschen. Diese veränderte Teilung von Entscheidungsgewalt und Verantwortung wird in den verschiedenen Funktionen der Organisation unterschiedlich sein. In diesem Beitrag wurde dies an Hand der Funktionsbereiche Führung, HR sowie Prozesse und Strukturen skizziert. Diese drei durchaus unterschiedlichen Bereiche wurden bewusst gewählt, um die Breite an Einsatzmöglichkeiten von AIS in Organisationen aufzuzeigen. Nur wenn AIS mit strategischen Zielen der Unternehmensführung, der Motivation und den Anforderungen von Mitarbeitern und organisationalen Prozessen ausgerichtet wird, ist von erfolgreichem Wirken auszugehen.

Auch sind die Grenzen fließend - je mehr sich die AIS weiterentwickeln und je mehr sie gerade auch in Kombination mit anderen Wissenschaften in der Lage ist, menschliches Agieren, Denken und Fühlen in Algorithmen abzubilden, desto mehr werden AIS Funktionen übernehmen, die bisher oder zum gegenwärtigen Zeitpunkt noch beim Menschen liegen. Die Frage der Verantwortungsübernahme für die getroffenen Entscheidungen wird derzeit intensiv weltweit in so unterschiedlichen Bereichen wie Informatik, Jura, Politik, Wirtschaftswissenschaften und Ethik diskutiert. Bekannt sind die Diskussionen v.a. im Kontext selbstfahrender Autos als Unfallverursacher (Brändle und Grundwald 2019). Entscheidend ist letztlich, ob AIS Autonomie und verantwortbares Risiko zugesprochen werden kann. Vertreter der Maschinenethik verfolgen den Ansatz, Maschinen so zu gestalten, dass sie als quasi-moralische Akteure akzeptiert werden; sehen aber die menschlichen Gestalter, Entwickler und Nutzer gleichzeitig als mitverantwortlich (Weber und Zoglauer 2019). Erklärbarkeit der AIS-Vorhersagen und erweiterte regulatorische Aufsicht sind weitere Aspekte, die bei der Gestaltung von AIS zu berücksichtigen sind. Der Anwender des AIS respektive der Bereitsteller des AIS muss verstehen können, warum die Entscheidung genauso erfolgte und wie man evtl. Fehler und Verzerrungen korrigieren kann. Die Arbeitsteilung zwischen Mensch und AIS erhält dadurch eine weitere Dimension. Die Implikationen für die existierende Arbeitswelt betreffen vor allem Fragen der Kompetenzentwicklung. Dies tangiert v. a. aber auch den grundsätzlichen Aspekt, was Arbeit zukünftig bedeutet, welchen Stellenwert Arbeit zukünftig hat und wie sich eine Gesellschaft und ein Arbeits-Leben darstellen, in dem Relevanz und Menge an Arbeit vielleicht abnehmen. Schon 1930 hat John Maynard Keynes den 3-Stunden-Arbeitstag für 2030 postuliert und die Gestaltung der Freizeit als größtes Problem angesehen (1930). Gleichzeitig bieten AIS Menschen völlig neue Möglichkeiten und Handlungsspielräume und eröffnen so neue Anwendungsfelder und Märkte. Welche Konsequenzen derartige Szenarien für den Wert von Arbeit für das Individuum, die Wertschätzung von Arbeit und Berufen in der Gesellschaft, die Finanzierung etc. haben, wird vielfach und kontrovers diskutiert.

Funding Open Access funding provided by Projekt DEAL.

Open Access Dieser Artikel wird unter der Creative Commons Namensnennung 4.0 International Lizenz veröffentlicht, welche die Nutzung, Vervielfältigung, Bearbeitung, Verbreitung und Wiedergabe in 
jeglichem Medium und Format erlaubt, sofern Sie den/die ursprünglichen Autor(en) und die Quelle ordnungsgemäß nennen, einen Link zur Creative Commons Lizenz beifügen und angeben, ob Änderungen vorgenommen wurden.

Die in diesem Artikel enthaltenen Bilder und sonstiges Drittmaterial unterliegen ebenfalls der genannten Creative Commons Lizenz, sofern sich aus der Abbildungslegende nichts anderes ergibt. Sofern das betreffende Material nicht unter der genannten Creative Commons Lizenz steht und die betreffende Handlung nicht nach gesetzlichen Vorschriften erlaubt ist, ist für die oben aufgeführten Weiterverwendungen des Materials die Einwilligung des jeweiligen Rechteinhabers einzuholen.

Weitere Details zur Lizenz entnehmen Sie bitte der Lizenzinformation auf http://creativecommons.org/ licenses/by/4.0/deed.de.

\section{Literatur}

Adler, R. 2019. Autonom oder vielleicht doch nur hochautomatisiert, was ist eigentlich der Unterschied? Fraunhofer IESE-Blog. https://blog.iese.fraunhofer.de/autonom-oder-vielleicht-doch-nurhochautomatisiert-was-ist-eigentlich-der-unterschied/. Zugegriffen: 6. Apr. 2020.

Ashton, K. 1999. That "Internet of Things" Thing. RFID Journal. June 2009. http://www.itrco.jp/libraries/ RFIDjournal-That\%20Internet\%20of\%20Things\%20Thing.pdf. Zugegriffen: 5. Aug. 2020.

Autor, D.H. 2015. Why are there still so many jobs? The history and future of workplace automation. Journal of Economic Perspectives 29(3):3-30.

Bainbrigde, L. 1983. Ironies of Automation. Automatica 19(6):775-779.

Bansal, M., A. Krizhevsky, und A. Ogale. 2018. Chauffeurnet: learning to drive by imitating the best and synthesizing the worst, arxiv:1812.03079. https://arxiv.org/pdf/1812.03079.pdf. Zugegriffen: 04.2020.

Benner, C. 2014. Crowd Work - Zurück in die Zukunft. Frankfurt: Bund.

Blier, N. 2020. Stories of AI failure and how to avoid similar AI fails. https://www.lexalytics.com/lexablog/ stories-ai-failure-avoid-ai-fails-2020. Zugegriffen: 04.2020.

Brändle, C., und A. Grundwald. 2019. Autonomes Fahren aus Sicht der Maschinenethik. In Handbuch Maschinenethik, Hrsg. O. Bendel. Wiesbaden: Springer.

Broussard, M. 2018. Artifical unintelligence-how computers misunderstand the world. Cambridge/ London: MIT Press.

Broussard, M. 2019. Statement by Meredith Broussard before the task force on artificial intelligence of the committee on financial services U.S. House of representatives. https://www.congress.gov/ 116/meeting/house/110094/witnesses/HHRG-116-BA00-Wstate-BroussardM-20191018.pdf. Zugegriffen: 18. Okt. 2019.

Brynjolfsson, E., und A. McAffee. 2014. The second machine age. Plassen: Kulmbach.

Brynjolfsson, E., und A. McAffee. 2017. Machine, platform, crowd. NewYork: Norton.

Busse, C. 2017. Adidas lässt Schuhe vom Roboter fertigen. In: Süddeutsche Zeitung. https://www. sueddeutsche.de/wirtschaft/sportartikel-adidas-laesst-schuhe-vom-roboter-fertigen-1.3693882. Zugegriffen: April 2020.

Chamorro-Premuzic, T., F. Polli, und B. Dattner 2019. Building Ethical AI for Talent Management. In: Harvard Business Review 11/2019. https://hbr.org/2019/11/building-ethical-ai-for-talent-management. Zugegriffen: September 2020.

Dastin, J. 2018. Amazon scraps secret AI recruiting tool that showed bias against women. Technology News, October 10, 2018. https://www.reuters.com/article/us-amazon-com-jobs-automation-insight/ amazon-scraps-secret-ai-recruiting-tool-that-showed-bias-against-women-idUSKCN1MK08G. Zugegriffen: April 2020.

Dietvorst, B., J. Simmons, und C. Massey. 2016. Overcoming algorithm aversion: people will use imperfect algorithms if they can (even slightly) modify them. Management Science 64(3):1155-1170. https:// doi.org/10.1287/mnsc.2016.2643.

Dietvorst, Berkeley, Joseph P. Simmons, und Cade Massey. 2014. Algorithm aversion: people erroneously avoid algorithms after seeing them err, forthcoming in journal of experimental psychology: general. https://ssrn.com/abstract=2466040 or https://doi.org/10.2139/ssrn.2466040 (Erstellt: July). Zugegriffen: April 2020. 
Domingos, P. 2015. The master algorithms how the quest for the ultimate learning machine remake our world. New York: Basic Books.

Dreyfus, H., und S. Dreyfus. 1988. Künstliche Intelligenz. Reinbek bei Hamburg: Rowohlt.

Dumitrescu, R., et al. 2018. Studie „Autonome Systeme“, Studien zum deutschen Innovationssystem. No. 13-2018. Berlin: Expertenkommission Forschung und Innovation (EFI). https://www.econstor.eu/ bitstream/10419/175555/1/1015315232.pdf. Zugegriffen: April 2020.

Ferràs-Hernández, X. 2018. The future of management in a world of electronic business. Journal of Management Inquiry 27(2):260-263.

Fesefeldt, J. 2018. Künstliche Intelligenz im Personalmanagement. DGP Informationen 68:6-37.

Fiedler, M. 2004. Expertise und Offenheit. Tübingen: Mohr Siebeck.

Fiedler, M., und E. Haruvy. 2016. The effect of third party intervention in the trust game. Journal of Behavioral and Experimental Economics 67:65-74.

Fiedler, M., E. Haruvy, und Sherry X. Li. 2011. Social distance in a virtual world experiment. Games and Economic Behavior 72(2):400-426.

Gatzke, M., L. Weigelin, B. Dibrani, J. Stork, H. Horn, und M. Schneider. 2018. Eckpunktepapier Künstliche Intelligenz und Maschinelles Lernen. Wuppertal: Bergische Universität Wuppertal.

Gierlich, M., T. Hess, und R. Neuburger. 2020. More self-organization, more control—or even both? Inverse transparency as a new digital leadership concept. Business Research. Forthcoming.

Glikson, E., und A. Woolley. 2020. Human trust in artificial intelligence: review of empirical research. Academy of Management Annals 14(2):627-660.

Gregory, R., O. Henfridsson, E. Kaganer, und H. Kyriakou. 2020. The role of artificial intelligence and data network effects for creating user value. Academy of Management Review. https://doi.org/10. 5465/amr.2019.0178.

Haase, T., W. Termath, und M. Schumann. 2015. Integrierte Lern-und Assistenzsysteme für die Produktion von morgen. In Lehren und Lernen für die moderne Arbeitswelt, 183-207. Berlin: Gito.

Hatmaker, T. 2020. AI researchers condemn predictive crime software, citing racial bias and flawed methods, Techcrunch, 24.Juni 2020. https://techcrunch.com/2020/06/23/ai-crime-prediction-open-letterspringer/?guccounter=1\&guce_referrer=aHR0cHM6Ly93d3cuZ29vZ2xlLmNvbS8\&guce_referrer_ sig=AQAAAB6Kw4H7siOhx6iodHDmWdGAjHqhnxFQX_eQ4cQY2J80MR3HTx8MEehGAHm NaoZ1BwDRnxpFbmMwItnipzD_cAwsr8KcRNWlArVam8A_YI7UsyFv-OLNMDgrWQqltR7MO ptrEXImnBu-2fbd3erVpwts4NSDVWEIuoHjXZgMFmBx. Zugegriffen: April 2020.

Hoffmeyer, M. 2020. Neuer Job per Chat. Nr. 80, 4./5.4.20, 59. In: Süddeutsche Zeitung. https://www. sueddeutsche.de/karriere/recruiting-neuer-job-per-chat-1.4864889. Zugegriffen: April 2020.

Hoßfeld, T., M. Hirth, und P. Tran-Gia. 2012. Aktuelles Schlagwort: Crowdsourcing. https://www.tuilmenau.de/fileadmin/media/mt-nam/media/publications/Aktuelles_Schlagwort_Crowdsourcing.pdf. Zugegriffen: 04.2020.

Ihl, Andreas, Kim Strunk, und Marina Fiedler. 2018. The influence of utilitarian and hedonic motivation on success in crowd work. Proceedings of the 39th International Conference on Information Systems (ICIS), San Francisco.

Ihl, A., K. Strunk, und M. Fiedler. 2020. The mediated effects of social support in professional online communities on crowdworker engagement in micro-task crowdworking. Computers in Human Behavior. https://doi.org/10.1016/j.chb.2020.106482.

Kaplan, A., und M. Haenlein. 2018. Siri, Siri, in my hand: Who's the fairest in the land? On the interpretations, illustrations, and implications of artificial intelligence. Business Horizons. https://doi.org/10. 1016/j.bushor.2018.08.004.

Keynes, J.M. 1930. Economic possibilities for our grandchildren. In Essays in persuasion, 358-373. New York: W.W. Norton \& Co..

Kilian, W., A. Picot, R. Neuburger, J. Niggl, K.-L. Scholtes, und W. Seiler. 1994. Electronic Data Interchange (EDI) aus ökonomischer und juristischer Sicht. Baden-Baden: Nomos.

Klausch, J. 2019. Wenn Roboter bei HR-Aufgaben unterstützen. Human Resources Manager. https://www. humanresourcesmanager.de/news/wenn-roboter-bei-hr-aufgaben-unterstuetzen-sponsored.html. Zugegriffen: April 2020.

Legal Tech. 2020. Die Digitalisierung des Rechtsmarkts. CH Beck. https://legalhead.de/blog/legal-techdie-digitalisierung-der-rechtsberatung/. Zugegriffen: April 2020.

Malone, T.W., R. Laubacher, und T. Johns. 2011. The big idea: the age of Hyperspecialization, Harvard Business Review, july-August 2011. https://hbr.org/2011/07/the-big-idea-the-age-of-hyperspeciali zation. Zugegriffen: April 2020.

McQuivey, J.L. 2014. Digital Disruption: Unleashing the next wave of innovation. https://pt.slideshare.net/ DelightConference/delight-2014?ref=. Zugegriffen: April 2020. 
Mertens, P. 1995. Wirtschaftsinformatik - Von den Moden zum Trend. In Wirtschaftsinformatik, Bd. 95, Hrsg. W. Wönig, 25-64. Heidelberg: Physica.

MÜNCHNER KREIS. 2020. Kompetenzentwicklung für und in der digitalen Arbeitswelt. Positionspapier des Arbeitskreises „Arbeit in der digitalen Welt“. München: MÜNCHNER KREIS. https:// www.muenchner-kreis.de/download/MUENCHNER-KREIS-Kompetenzpapier.pdf. Zugegriffen: Juli 2020 .

Obermaier, R. 2019. Handbuch Industrie 4.0 und Digitale Transformation. Betriebswirtschaftliche, technische und rechtliche Herausforderungen. Wiesbaden: Springer.

Obermeyer, Z., B. Powers, C. Vogeli, und S. Mullainathan. 2019. Dissecting racial bias in an algorithm used to manage the health of populations. Science 366(6464):447-453.

Peissner, M., F. Kötter, und H. Zaiser. 2019. Künstliche Intelligenz - Anwendungsperspektiven für Arbeit und Qualifizierung. In Digitalisierung und Künstliche Intelligenz, Hrsg. BWP Berufsbildung in Wissenschaft und Praxis, 9-13. Stuttgart: Franz Steiner.

Picot, A. 2016. Digitalisierung der Arbeitswelt: Zwischen Flexibilisierung und Polarisierung? Vortrag Bayerische Akademie der Wissenschaften, München.

Picot, A., und R. Neuburger. 2020. Kompetenzen für Big Data aus Unternehmenssicht. In Big Data und Mittelstand - Ergebnisse des ABIDA-Projektes, Hrsg. A. Picot, R. Neuburger, Y. Berchtold, und A. Defort. Wiesbaden: Springer.

Picot, A., H. Dietl, E. Franck, M. Fiedler, und S. Royer. 2020a. Organisation-Theorie und Praxis aus ökonomischer Sicht, 8. Aufl., Stuttgart: Schäffer-Poeschel.

Picot, A., R. Reichwald, R.T. Wigand, K.M. Möslein, R. Neuburger, und A.-K. Neyer. 2020b. Die grenzenlose Unternehmung. Information, Organisation \& Führung. Wiesbaden: Springer.

Polanyi, Michael. 1966. The tacit dimension. New York: Garden City. deutsch: Implizites Wissen, Frankfurt am Main, 1985.

Polanyi, Michael, und Harry Prosch. 1975. Meaning, chicago, IL, 1975.Polli, F. 2019. Using AI to eliminate bias from hiring. Harvard Business Review, October 29 2019. https://hbr.org/2019/10/using-aito-eliminate-bias-from-hiring. Zugegriffen: April 2020.

Polanyi, Michael. 1957. Problem solving. The british journal for the philosophy of science. https://doi.org/ 10.2307/685789.

Rai, A., P. Constantinides, und S. Sarker. 2019. Next-generation digital platforms: toward human-AI hybrids. MIS Quarterly 43(1):iii-ix.

Raisch, S., und S. Krakowski. 2020. Artificial intelligence and management: the automation-augmentation paradox. Academy of Management Review https://doi.org/10.5465/2018.0072.

Ramaprasad, J., K. Ganju, und T. Singh. 2020. AI and decision making: programming, biases, and moral decision-making. Präsentation im Rahmen des Symposiums „Broadening our Sight to Artificial Intelligence in Management (session 45), Academy of Management Conference 2020".

Ross, C., und I. Swetlitz. 2018. IBM's Watson supercomputer recommended "unsafe and incorrect" cancer treatments, internal documents show. StatNews, July 25, 2018. https://www.statnews.com/2018/07/ 25/ibm-watson-recommended-unsafe-incorrect-treatments/. Zugegriffen: April 2020.

Russel, S., und P. Norvig. 2010. Artificial intelligence-A modern approach, 3. Aufl., Essex: Pearson.

SAE. 2019. Taxonomy and definitions for terms related to driving automation systems for on-road motor vehicles. SAE international. https://www.sae.org/standards/content/j3016/. Zugegriffen: April 2020.

Sattelberger, T. 2016. Skillshift: Quantensprung in Qualität und Quantität. Vortragsunterlagen zum Berliner Gespräch des MÜNCHNER KREIS am 18.4.2018.

Scheelen, F. 2019. Algorithmen übernehmen: Führung mittels Künstlicher Intelligenz. CIO von IDG. https://www.cio.de/a/fuehrung-mittels-kuenstlicher-intelligenz,3583807. Zugegriffen: April 2020.

Stahlknecht, P. 1982. EDV-Systeme im Finanz- und Rechnungswesen: Anwendergespräch. Osnabrück: Springer.

Strickland, E. 2019. How IBM Watson Overpromised and Underdelivered on AI Health Car. IEEE Spectrum. https://spectrum.iee.org/biomedical/diagnostics/how-ibm-watson-overpromised-andunderdelivered-on-ai-health-care. Zugegriffen: April 2020.

Tang, Y., C. Zhao, J. Wang, C. Zhang, Q. Sun, W.X. Zheng, W. Du, F. Qian, und J. Kurths. 2020. An overview of perception and decision-making in autonomous systems in the era of learning. https:// arxiv.org/pdf/2001.02319.pdf. Zugegriffen: April 2020.

Vogl, E. 2018. Crowdsourcing-Plattformen als neue Marktplätze für Arbeit: Die Neuorganisation von Arbeit im Informationsraum und ihre Implikationen. Augsburg: Hampp.

Weber, S. 2019. Roboterjournalismus, Chatbots \& Co.: Wie Algorithmen Inhalte produzieren und unser Denken beeinflussen. Hannover: Heise Gruppe. 
Weber, K., und T. Zoglauer. 2019. Maschinenethik und Technikethik. In Maschinenethik, Hrsg. O. Bendel, 145-163. Wiesbaden: Springer.

Welsch, A., V. Eitle, und P. Buxmann. 2018. Maschinelles Lernen - Grundlagen und betriebswirtschaftliche Anwendungspotenziale am Beispiel von Kundenbindungsprozessen. HMD 55:366-382.

Wilkens, R., und R. Falk. 2019. Smart Contracts: Grundlagen, Anwendungsfelder und rechtliche Aspekte. Berlin: Springer.

Wirtz, B.W. 2018. Electronic business. Berlin: Springer.

Writer, B. 2019. Lithium-Ion batteries: a machine-generated summary of current research. Wiesbaden: Springer.

Yeomans, M., A. Shah, S. Mullainathan, und J. Kleinberg. 2019. Making sense of recommendations. Journal of Behavioral Decision Making 32(4):403-414.

Zuboff, S. 1988. In the age of the smart machine: The future of work and power. New York: Basic Books. 